Canad. Math. Bull. Vol. 17 (3), 1974

\title{
THE LOCAL GROWTH OF POWER SERIES: A SURVEY OF THE WIMAN-VALIRON METHOD
}

BY

W. K. HAYMAN

\section{CONTENTS}

$\begin{array}{ll}\text { 1. Introduction and terminology } & 317\end{array}$

$\begin{array}{lll}1.1 & \text { Densities } & 318\end{array}$

$\begin{array}{lll}1.2 & \text { The basic results } & 319\end{array}$

2. Construction of the comparison sequences 321

2.1 Estimates for the terms of integral functions 322

3. The truncated series 324

3.1 Estimates for the terms far from the maximum term 326

$\begin{array}{lll}3.2 & \text { Similar estimates for functions of order }(\log r)^{2} & 328\end{array}$

3.3 Functions of order $(\log r)^{p}$, when $p<2 \quad 328$

4. Maximum modulus and maximum term 330

$\begin{array}{lll}\text { 4.1 The main results } & 333\end{array}$

5. The minimum modulus of functions of small growth 334

6. Behaviour near the local maximum 336

6.1 Statement and proof of the main results 338

$\begin{array}{ll}\text { 6.2 Behaviour of derivatives } & 341\end{array}$

6.3 A generalization of Picard's theorem 343

7. Two Counter-examples: The sigma function 345

7.1 How large must $k$ and $\eta$ be? Another example 347

8. Maximum and minimum of the real part 349

8.1 Proof of Theorem 15: preliminaries 350

8.2 Completion of proof of Theorem $15 \quad 353$

8.3 Proof of Theorem $16 \quad 354$

9. Conclusion $\quad 357$

$\begin{array}{ll}\text { References } & 357\end{array}$

1. Introduction and terminology. Suppose that

$$
f(z)=\sum_{0}^{\infty} a_{n} z^{n}=u+i v
$$

is a transcendental integral function. In this article we develop the theory initiated

Received by the editors April, 1972 
by Wiman $[22,23]$ and deepened by other writers including Valiron $[18,19,20]$, Saxer [15], Clunie [4, 5] and Kövari [10, 11], which describes the local behaviour of $f(z)$, near a point where $|f(z)|$ is large, in terms of the power series $\dagger$ of $f(z)$. The theory leads in particular to a good account of the relation between the maximum modulus

and the maximum term

$$
M(r, f)=\sup _{|z|=r}|f(z)|
$$

$$
\mu(r)=\sup _{n}\left|a_{n}\right| r^{n}=a_{N} r^{N}
$$

say. Here $N=N(r)$ is called the central index. If there are several maximum terms, which can only happen for isolated values of $r$ we define $N(r)$ to be the largest of the corresponding indices. The theory also allows us to compare

$$
A(r)=\inf _{|z|=r} u(z) \text { and } \quad B(r)=\sup _{|z|=r} u(z)
$$

We recall that $\log M(r)$ is an increasing convex function of $\log r$. Thus

$$
a(r)=r \frac{d}{d r} \log M(r), \quad \text { and } \quad b(r)=r \frac{d}{d r} a(r)
$$

exist except for isolated values of $r$ and $a(r)$ increases with $r$, while $b(r) \geq 0$.

We note that $N(r)$ is an increasing function of $r$ and $N(r) \rightarrow \infty$ with $r$. If

$$
N(r)=N \text { for } r_{N} \leq r<r_{N+1}
$$

we see that

$$
r \frac{d}{d r} \log \mu(r)=N=N(r), \quad r_{N}<r<r_{N+1} .
$$

Since $\mu(r)$ is continuous at the points $r_{N}$, we deduce that

$$
\log \mu(r)=\log \mu\left(r_{0}\right)+\int_{r_{0}}^{r} \frac{N(t) d t}{t}, \quad 0<r_{0}<r<\infty .
$$

Thus $\log \mu(r)$ is also a convex increasing function of $\log r$.

1.1. Densities. In order to develop our results we shall need various kinds of measures and densities for sets of points on the positive axis. Let $E$ be such a set and let $E[a, b]$ denote the part of $E$ for which $a<r<b$. The linear and logarithmic measures of $E$ are defined to be

$$
m(E)=\int_{E} d r \quad \text { and } \quad \operatorname{lm}(E)=\int_{E(1, \infty)} \frac{d r}{r}
$$

respectively. These may be finite or infinite. We also define the lower and upper

$\dagger$ For an extension of the theory to functions in a finite disk see [12]. 
densities of $E$ by

$$
\overline{\mathrm{dens}} E=\varlimsup_{r \rightarrow \infty} \frac{m(E(0, r))}{r}
$$

and the upper and lower logarithmic densities of $E$ by

$$
\overline{\log \operatorname{dens}} E=\varlimsup_{r \rightarrow \infty} \frac{\operatorname{lm}(E(1, r))}{\log r} .
$$

The definition (1.3) only tells us that the term with index $N$ equal to the central index is larger than any of the others in the power series (1.1). The key result of the Wiman-Valiron Theory allows us to obtain a definite inequality for

$$
\frac{\left|a_{n}\right| r^{n}}{\mu(r)}
$$

when $n$ is unequal to $N$, which is valid for all $n$ and 'most' values of $r$ in a certain sense and shows that the terms for which $|n-N| \geq k$ are negligible compared with $\mu(r)$ if for instance $k \geq N^{1 / 2+\delta}$. This reduces the local behaviour of $f(z)$ essentially to that of $z^{N-k} \mathrm{P}(z)$, where $\mathrm{P}(z)$ is a polynomial of degree at most $2 k$.

1.2. The basic results. We now introduce the comparison series. Let $\alpha_{n}, n=0$ to $\infty$ be a sequence of positive numbers such that $\alpha_{n+1} / \alpha_{n}$ decreases with increasing $n$. Let $\rho_{n}$ be a sequence of numbers such that

$$
0<\rho_{0}<\frac{\alpha_{0}}{\alpha_{1}}, \quad \frac{\alpha_{n-1}}{\alpha_{n}}<\rho_{n}<\frac{\alpha_{n}}{\alpha_{n+1}}, \quad n \geq 1
$$

so that $\rho_{n}$ increases with increasing $n$. We shall say that a value $r$ is normal (for the sequences $a_{n}, \rho_{n}$ and $\alpha_{n}$ ) if we have for some $N$

$$
\left|a_{n}\right| r^{n} \leq\left|a_{N}\right| r^{N} \frac{\alpha_{n} \rho_{N}^{n}}{\alpha_{N} \rho_{N}^{N}}, \quad n=0 \text { to } \infty .
$$

It follows from the definition of $\rho_{N}$ that for $n \neq N$

$$
\frac{\alpha_{n}}{\alpha_{N}}<\rho_{N}^{N-n}
$$

so that if (1.10) holds, $N=N(r)$ is necessarily the central index of $f(z)$. It turns out that, the more rapidly $\alpha_{n}$ tends to zero, the stronger on the whole is the information contained in (1.10), but the smaller is the set of normal $r$. The set of $r$ which are not normal will be called exceptional.

The following result gives us information about the size of the set of normal $r$ in different situations.

THEOREM 1. Suppose that $r_{N}$ is defined by (1.6) and that $\rho_{N}$ satisfies (1.9). Then

(i) If $\rho_{N}$ is bounded above the set of exceptional $r$ has finite logarithmic measure; 
(ii) if

$$
\varlimsup_{N \rightarrow \infty} \frac{\log \rho_{N+1}}{\log r_{N}}=\delta<1
$$

then the set of exceptional $r$ has upper logarithmic density at most $\delta$.

(iii) If $\rho_{N} / r_{N} \rightarrow 0$ as $N \rightarrow \infty$, the set of normal $r$ has infinite logarithmic measure.

If $N(r)$ jumps from $N$ to $N+k$ as $r$ increases through the value $r_{N+1}$, we define $r_{N+1}=r_{N+2}=\cdots=r_{N+k}$. With this definition we see that

so that

$$
\left|a_{N+k}\right| r_{N+k}^{N+k}=\left|a_{N}\right| r_{N+1}^{N}
$$

and

$$
\left|a_{N+k}\right|=\frac{\left|a_{N}\right|}{r_{N+1}^{k}}=\frac{a_{N}}{r_{N+1} \cdots r_{N+k}},
$$

Thus we always have

$$
\left|a_{N+v}\right| \leq \frac{\left|a_{N}\right|}{r_{N+1}^{v}}, \quad 1 \leq v \leq k
$$

$$
\frac{\left|a_{N}\right|}{\left|a_{0}\right|} \leq \frac{1}{r_{1} r_{2} \cdots r_{N}} .
$$

On the other hand we deduce from (1.9) that

so that

$$
\frac{\alpha_{n}}{\alpha_{n-1}} \geq \frac{1}{\rho_{n}}
$$

In particular

$$
\frac{\alpha_{N}}{\alpha_{0}} \geq \frac{1}{\rho_{1} \rho_{2} \cdots \rho_{N}}
$$

$$
\left(\frac{a_{N}}{\alpha_{N}}\right)^{1 / N} \leq\left(\frac{a_{0}}{\alpha_{0}} \frac{\rho_{1}}{r_{1}} \ldots \frac{\rho_{N}}{r_{N}}\right)^{1 / N} \rightarrow 0 \text { as } \quad N \rightarrow \infty
$$

even under the weakest hypothesis (iii) of Theorem 1 . Thus if any of the hypotheses (i), (ii) or (iii) of that theorem hold, and we set

it follows that

$$
A_{N}=\left|\frac{a_{N}}{\alpha_{N}}\right|
$$

$$
F(z)=\sum_{0}^{\infty} A_{n} z^{n}
$$

is an integral function. It follows that for $0<\rho<\infty, F(z)$ has a maximum term for $|z|=\rho$, and if $M=M(\rho)$ is the central index of $F(z)$ for $|z|=\rho$, we deduce that

$$
A_{n} \rho^{n} \leq A_{M} \rho^{M}, \quad n=0 \text { to } \infty \text {. }
$$


The inequality can be written as

$$
\frac{\left|a_{n}\right|\left(\rho \rho_{M}\right)^{n}}{\left|a_{M}\right|\left(\rho \rho_{M}\right)^{M}} \leq \frac{\alpha_{n} \rho_{M}^{n}}{\alpha_{M} \rho_{M}^{M}}<1, \quad n \neq N
$$

in view of (1.11). Thus if we set $r=\rho \rho_{M}$, it follows that $N(r)=M$, and the inequality (1.10) holds. Thus $r$ is normal if $r$ takes this form, i.e. if we can write $r=\rho \rho_{M}$, where $M$ is the central index of $F(z)$ for $|z|=\rho$.

Let $\left(R_{n}, R_{n+1}\right)$ be the interval in which the central index of $F(z)$ is $n$, so that $R_{n}$ is defined for $F(z)$ as $r_{n}$ is defined for $f(z)$ by (1.6). Then the intervals $\left(R_{n} \rho_{n}, R_{n+1} \rho_{n}\right)$ are normal so that the exceptional values of $r$ are contained in the complementary intervals of the form $\left(R_{n} \rho_{n-1}, R_{n} \rho_{n}\right)$. Let $E_{N}$ be the set of these complementary intervals for $n=1$ to $N$. Then

$$
\operatorname{lm}\left(E_{N}\right) \leq \sum_{n=1}^{N} \int_{R_{n} \rho_{n-1}}^{R_{n} \rho_{n}} \frac{d t}{t}=\log \frac{\rho_{N}}{\rho_{0}}
$$

Also the exceptional $r$ for $r \leq R_{N+1} \rho_{N}$ are contained in $E_{N}$.

Next we recall that the central index of $f(z)$ is $n$ in the interval $\left(\rho_{n} R_{n}, \rho_{n} R_{n+1}\right)$, so that $r_{n} \leq \rho_{n} R_{n}$. Thus if $r_{n} \leq r \leq r_{n+1}$, we deduce that $r \leq \rho_{n+1} R_{n+2}$, so that the exceptional $t$ in $(0, r)$ are contained in $E_{n+1}$ and so have logarithmic measure at most $\log \left(\rho_{n+1} / \rho_{0}\right)$. Thus if $E$ is the set of exceptional $t$, we have

$$
\operatorname{lm}(E(1, r)) \leq \log \frac{\rho_{n+1}}{\rho_{0}}, \quad r_{n} \leq r \leq r_{n+1} .
$$

Now Theorem 1 follows at once. If $\rho_{N}$ is bounded above then so is $\operatorname{lm}(E(1, r))$ as $r \rightarrow \infty$, so that $E$ has finite logarithmic measure. If (ii) holds then

$$
\frac{\operatorname{lm}(E(1, r))}{\log r} \leq \frac{\log \rho_{n+1}+O(1)}{\log r} \leq \frac{\log \rho_{n+1}+O(1)}{\log r_{n}} \leq \delta+o(1), \text { as } n \rightarrow \infty,
$$

so that our conclusion follows again. Finally if (iii) holds then

$$
\operatorname{lm}\left(E\left(1, r_{n+1}\right)\right) \leq \log \rho_{n+1}+O(1),
$$

so that if $E^{\prime}$ is the set of normal $r$, we have

$$
\operatorname{lm}\left(E^{\prime}\left(1, r_{n+1}\right)\right) \geq \log \frac{r_{n+1}}{\rho_{n+1}}+O(1) \rightarrow \infty, \text { as } n \rightarrow \infty .
$$

Thus in this case $E^{\prime}$ must have infinite logarithmic measure. This completes the proof of Theorem 1 .

2. Construction of the comparison sequences. We now proceed to give a general method due to Kövari [11], for constructing the sequences $\alpha_{n}$, which makes it easy to estimate the quantities occurring on the right hand side of (1.10). Such estimates are fundamental to the Wiman-Valiron method. 
Suppose then that $\alpha(t) \in C^{1}[0, \infty)$ and that $\alpha^{\prime}(r)<0$ there. We set

$$
\alpha_{n}=K \exp \left\{\int_{0}^{n} \alpha(t) d t\right\}, \quad \rho_{n}=\exp \{-\alpha(n)\},
$$

where $K$ is a positive constant. Then for $n \geq 1$

$$
\log \frac{\alpha_{n}}{\alpha_{n-1}}=\int_{n-1}^{n} \alpha(t) d t>\alpha_{n}=\log \frac{1}{\rho_{n}}>\int_{n}^{n+1} \alpha(t) d t=\log \frac{\alpha_{n+1}}{\alpha_{n}},
$$

and the right hand inequality remains valid for $n=0$. This proves (1.9). Again

$$
\begin{aligned}
\frac{\alpha_{n}}{\alpha_{M}} \rho_{M}^{n-M} & =\exp \left\{\int_{M}^{n}(\alpha(t)-\alpha(M)) d t\right\} \\
& =\exp \left\{\int_{M}^{n}(n-t) \alpha^{\prime}(t) d t\right\} .
\end{aligned}
$$

Thus we deduce that for $|n-M| \geq k$, we have

$$
\frac{\alpha_{n}}{\alpha_{M}} \rho_{M}^{n-M} \leq \exp \left\{-\frac{1}{2} k^{2} \alpha_{M, k}\right\}
$$

where

$$
\alpha_{M, k}=\min _{|M-t| \leq k}\left|\alpha^{\prime}(t)\right| .
$$

2.1. Estimates for the terms of integral functions. By specialising the function $\alpha(t)$ we can obtain a number of results adapted to particular situations.

THEOREM 2. If $f(z)$ is any integral function we have for normal $r$ and $n=k+N$, where $N=N(r)$ is the central index of $f(z)$,

$$
\frac{\left|a_{n}\right| r^{n}}{\mu(r)} \leq \exp \left\{-\frac{1}{2} b(|k|+N) k^{2}\right\}
$$

where $\quad b(N)^{-1}=K N \log N \cdots \log _{l} N\left(\log _{l+1} N\right)^{1+\delta}, K>0, \log _{l} x$ iterated denotes the l times iterated logarithm, $\delta>0$ and the set of exceptional $r$ has finite logarithmic measure.

If we have some more information about the size of $f(z)$ we can obtain (2.4) with a larger value of $b(|k|+N)$. We give two further examples.

THEOREM 3. Suppose that $f(z)$ has finite order $\rho$. Then for $c<1 / \rho(2.4)$ holds with $b(N)=c / N$. The corresponding set of exceptional $r$ has upper logarithmic density at most $c \rho$.

$\uparrow$ This function is not to be confused with that defined by (1.5). If $b(r)$ is defined by (1.5) this will always be stated explicitly. Somewhat more general test functions were used by Kövari [11]. 
THEOREM 4. Suppose that for some constants $p \geq 2$ and $c>0$, we have

$$
\varlimsup_{r \rightarrow \infty} \frac{\log M(r, f)}{(\log r)^{p}} \leq c,
$$

and take $\sigma$ so that $\delta=\sigma(c p e)^{1 /(p-1)}<1$. Then for normal $r$ we have (2.4) with $b(N)=\sigma(p-1)^{-1} N^{(2-p) /(p-1)}$ and the set of exceptional $r$ has upper logarithmic density at most $\delta$.

A corresponding result for $p<2$ can be deduced but turns out to be less useful. The above results can all be deduced from (2.1) and (2.2) with a suitable choice of the function $\alpha(t)$. For Theorem 2 we take

$$
\begin{aligned}
& \alpha^{\prime}(t)=-\frac{1}{K t \log t \cdots \log _{l} t\left(\log _{l+1} t\right)^{1+\delta}}, \quad t \geq t_{0} \\
& \alpha^{\prime}(t)=\alpha^{\prime}\left(t_{0}\right), \quad t \leq t_{0},
\end{aligned}
$$

where $t_{0}$ is a sufficiently large positive number and set

$$
\alpha(t)=\int_{0}^{t} \alpha^{\prime}(\tau) d \tau
$$

Then $\alpha(t)$ is negative decreasing and bounded below as $t \rightarrow \infty$. Thus we can apply Theorem 1 and note that the exceptional set of $r$ has finite logarithmic measure. Also $\left|\alpha^{\prime}(t)\right|$ decreases with $t$, so that

$$
\alpha_{M, k}=\min _{|M-t| \leq k}\left|\alpha^{\prime}(t)\right|=-\alpha^{\prime}(M+|k|) .
$$

Now (2.4) follows from (1.10), (2.2) and (2.3).

Next in the case of Theorem 3 , we choose

$$
\begin{array}{ll}
\alpha^{\prime}(t)=\frac{-c}{t}, & t \geq 1, \\
\alpha^{\prime}(t)=-c, \quad t \leq 1,
\end{array}
$$

so that $\alpha(t)=-c \log t, t \geq 1$, and then (2.1) with a suitable $K$ gives

$$
\rho_{n}=n^{c}, \quad \alpha_{n}=\exp \left\{-c \int_{0}^{n} \log t d t\right\}=(e / n)^{c n}, \quad n \geq 1 .
$$

Also by our hypotheses we have for $\rho_{1}>\rho$

$$
\log M(r, f)<\left(\frac{r}{e}\right)^{\rho_{1}}, \quad r>r_{0}
$$

and so we have by (1.8),

$$
\begin{aligned}
N(r) & \leq \int_{r}^{e r} \frac{N(t) d t}{t} \leq \log \mu(e r) \\
& \leq \log M(e r)<r^{\rho_{1}}, \quad r>r_{0},
\end{aligned}
$$


so that $r_{N} \geq N^{1 / \rho_{1}}$. This gives for all sufficiently large $N$

$$
\frac{\log \rho_{n+1}}{\log r_{n}} \leq c \rho_{1} \frac{\log (n+1)}{\log n},
$$

and since $\rho_{1}$ may be chosen as near $\rho$ as we please, we may apply Theorem 1 case (ii) with $\delta=c \rho$. Thus the set of exceptional $r$ has upper logarithmic density at most $c \rho$.

We now note that $\left|\alpha^{\prime}(t)\right|$ decreases for $t \geq 0$ so that we have

$$
\alpha_{M, k} \geq\left|\alpha^{\prime}(M+|k|)\right|=c /(M+|k|) .
$$

Now Theorem 3 follows from (1.10), (2.2) and (2.3).

Finally we suppose that $f(z)$ satisfies the hypotheses of Theorem 4 . We set $k=1+p^{-1}$, and deduce that for $c^{\prime}>c$ and $r$ large, we have,

Thus

$$
\begin{aligned}
(k-1) N(r) \log r & \leq \int_{r}^{r^{k}} N(t) \frac{d t}{t} \leq \log \mu\left(r^{k}\right) \\
& \leq \log M\left(r^{k}\right)<c^{\prime} k^{p}(\log r)^{p} .
\end{aligned}
$$

$$
N(r)<c^{\prime} p\left(1+p^{-1}\right)^{p}(\log r)^{p-1}<c e p(\log r)^{p-1}
$$

if $c^{\prime}$ is sufficiently near $c$. Thus

$$
r_{N}>\exp (N / c p e)^{1 /(p-1)} .
$$

We now assume that $\sigma(c p e)^{1 /(p-1)}=\delta<1$, and set

so that (2.1) gives

$$
\alpha(t)=-\sigma t^{1 /(p-1)},
$$

and we deduce that

$$
\log \rho_{N}=\sigma N^{1 /(p-1)},
$$

$$
\varlimsup_{N \rightarrow \infty} \frac{\log \rho_{N+1}}{\log r_{N}} \leq \sigma(c p e)^{1 /(p-1)}=\delta .
$$

We can then apply (2.2) with (2.3). Also, since $p \geq 2,\left|\alpha^{\prime}(t)\right|$ decreases with increasing $p$, and so we have again

and we deduce Theorem 4.

$$
\alpha_{M, k}=\left|\alpha^{\prime}(M+|k|)\right|,
$$

3. The truncated series. We proceed to show that for normal $|z|$ and large $|f(z)|$ only a few terms in the neighbourhood of the maximum term are of genuine significance in the power series development of $f(z)$. We need first a general lemma on the growth of increasing functions.

Lemma 1. Suppose that $N(r)$ is a positive increasing function of $r$ for $r \geq r_{0}$. Then if $\alpha>0$, and $|h|<N(r)^{-\alpha}$, we have

$$
\left|N\left(r e^{h}\right)-N(r)\right|<\alpha N(r)
$$

for all $r$ outside a set of finite logarithmic measure. 
We shall assume that $N(r)$ is continuous on the right. For otherwise we may achieve this by altering $N(r)$ at a countable set of $r$ and this will not affect the truth of (3.1) at other points.

For each $r \geq r_{0}$, we define $h(r)=N(r)^{-\alpha}$. We then define sequences $r_{n}, r_{n}^{\prime}$ inductively as follows. Let $r_{0}$ be the quantity of Lemma 1 . If $r_{n}$ has already been defined we define

$$
r_{n}^{\prime}=r_{n} \exp h\left(r_{n}\right), \quad r_{n}^{\prime \prime}=r_{n}^{\prime} \exp h\left(r_{n}\right)
$$

and $r_{n+1}$ to be the least number $\dagger$ (if any) such that $r_{n+1} \geq r_{n}^{\prime}$ and

$$
N\left\{r_{n+1} \exp h\left(r_{n+1}\right)\right\} \geq(1+\alpha) N\left(r_{n+1}\right) .
$$

Evidently we have for $n \geq 1$, if $r_{n}^{\prime}$ is defined,

$$
N\left(r_{n}^{\prime}\right) \geq(1+\alpha) N\left(r_{n}\right) \geq(1+\alpha) N\left(r_{n-1}^{\prime}\right) \geq \ldots \geq(1+\alpha)^{n} N\left(r_{0}\right) .
$$

Thus

$$
\begin{aligned}
\sum_{1}^{\infty} \log \left(\frac{r_{n}^{\prime \prime}}{r_{n}}\right) & =2 \sum_{1}^{\infty} h\left(r_{n}\right)=2 \sum_{1}^{\infty} N\left(r_{n}\right)^{-\alpha} \\
& \leq 2 \sum_{1}^{\infty}(1+\alpha)^{-\alpha(n-1)} N\left(r_{0}\right)^{-\alpha}<\infty,
\end{aligned}
$$

so that the union $E_{0}$ of all the intervals $\left[r_{n}, r_{n}^{\prime \prime}\right]$ has finite logarithmic measure.

Let $E_{1}$ be the complement of $E_{0}$ in $\left(r_{0}, \infty\right)$ and suppose that $r \in E_{1}$. Then either $r_{n}$ is undefined for large $n$, in which case we have for all sufficiently large $r$

$$
N\{r \exp h(r)\}<(1+\alpha) N(r) ;
$$

also if $r^{\prime}=r \exp h(r)$, we have

$$
r^{\prime} \exp -h\left(r^{\prime}\right) \geq r^{\prime} \exp -h(r)=r,
$$

so that for all large $r^{\prime}$ we have

$$
N\left\{r^{\prime} \exp -h\left(r^{\prime}\right)\right\} \geq N(r) \geq \frac{1}{1+\alpha} N\left(r^{\prime}\right) \geq(1-\alpha) N\left(r^{\prime}\right) .
$$

Thus in this case (3.1) holds for all sufficiently large $r$.

Alternatively suppose that $r_{n}$ is defined for all $n$, and let $r$ be a point of $E_{1}$. Then we have $r_{n}^{\prime}<r<r_{n+1}$ for some $n$, so that (3.2) holds. Also suppose that

$$
N\left(r e^{-h(r)}\right) \leq(1-\alpha) N(r)
$$

Then if $\rho=r \exp (-h(r))$, we have

so that

$$
\rho e^{h(\rho)} \geq \rho e^{h(r)}=r
$$

$$
N\left(\rho e^{h(\rho)}\right) \geq \frac{1}{1-\alpha} N(\rho)>(1+\alpha) N(\rho)
$$

$\dagger$ The fact that the lower bound is attained follows from the continuity on the right of $N(r)$. 
Thus $\rho$ must lie in one of the intervals $\left[r_{v}, r_{v}^{\prime}\right]$ and so

$$
r=\rho e^{h(r)} \leq \rho e^{h\left(r_{v}\right)} \leq r_{v}^{\prime} e^{h\left(r_{v}\right)}=r_{v}^{\prime \prime},
$$

so that $r$ lies in $E_{0}$ contrary to hypothesis. Thus (3.3) must be false and so (3.1) holds for $r$ in $E_{1}$ and $h=\mp h(r)$. Since $N(r)$ increases with $r$, (3.1) also holds for $|h| \leq h(r)$ and Lemma 1 is proved.

From now on we shall call normal those values of $r$ which satisfy (3.1) for a suitable constant $\alpha$ as well as (1.12). Since the exceptional set in (3.1) has finite logarithmic measure, the conclusions of Theorem 1 to 4 will remain with this restricted notion of normality.

3.1 Estimates for the terms far from the maximum term. Our main result in this section is

Lemma 2. Suppose that $r$ is normal in the sense of Theorems 2 or 3 or the case $p>2$ of Theorem 4. We write $N=N(r)$ and suppose that (3.1) holds with a suitable $\alpha$. Suppose that $\gamma$ is a positive constant and write

$$
k=\left[\left\{\frac{\gamma}{b(N)} \log \frac{1}{b(N)}\right\}^{1 / 2}\right]
$$

where $[x]$ denotes the integral part of $x$. Further suppose that

$$
|\log (\rho / r)| \leq 2 k^{-1} \text {, and write } \mu_{0}(\rho)=\left|a_{N}\right| \rho^{N} .
$$

Then we have for any fixed real $q$ and $\gamma_{1}<\gamma$

$$
\sum_{|n-N| \geq k} n^{\alpha}\left|a_{n}\right| \rho^{n}=o\left\{\mu_{0}(\rho) N^{\alpha} b(N)^{(1 / 2) \gamma_{1}-(1 / 2)}\right\}
$$

uniformly as $\rho$ and $r$ tend to infinity subject to the above inequalities.

Let $\eta$ be a constant, $0<\eta \leq \frac{1}{2}$, and assume first that $n \geq(1+\eta) N$. We choose a fixed $\alpha \leq \frac{1}{2} \eta$ in Lemma 1 and write

$$
\rho_{1}=r \exp \left(N^{-\alpha}\right) .
$$

Let $M$ be the central index for $\rho_{1}$, so that we have by Lemma 1

$$
N \leq M \leq(1+\alpha) N, \text { and }\left|a_{n}\right| \rho_{1}^{n} \leq\left|a_{M}\right| \rho_{1}^{M} .
$$

Thus $n-M \geq \frac{1}{2} \eta n$. Also

$$
\begin{aligned}
\frac{\left|a_{n}\right| \rho^{n}}{\mu_{0}(\rho)} & =\frac{\left|a_{n}\right| \rho^{n}}{\left|a_{N}\right| \rho^{N}}=\frac{\left|a_{n}\right| \rho^{n}}{\left|a_{M}\right| \rho_{1}^{M}} \cdot \frac{\left|a_{M}\right| r^{M}}{\left|a_{N}\right| r^{N}} \cdot\left(\frac{\rho_{1}}{r}\right)^{M-n} \cdot\left(\frac{\rho}{r}\right)^{n-N} \\
& \leq\left(\frac{\rho_{1}}{r}\right)^{M-n}\left(\frac{\rho}{r}\right)^{n-N} \leq \exp \left\{-\frac{1}{3} \eta n N^{-\alpha}+n|\log (\rho / r)|\right\} .
\end{aligned}
$$

From the hypotheses of Lemma 2 it follows that

$$
|\log (\rho / r)| \leq 2 k^{-1} \leq o\left\{N^{-1 / 4}\right\}
$$


with the hypotheses of Theorem 2 and 3 and

$$
k^{-1}=o\left\{N^{(2-p) /[3(p-1)]}\right\}
$$

in the case of Theorem 4. By choosing

$$
\alpha=\frac{1}{3} \frac{p-2}{p-1}, \quad \text { or } \quad \alpha=\frac{1}{2} \eta,
$$

we can thus ensure that when $r$ and $N$ are large

$$
\frac{\left|a_{n}\right| \rho^{n}}{\mu_{0}(\rho)} \leq \exp \left\{-\frac{\eta n}{6} N^{-\alpha}\right\} .
$$

We now set $t=\exp \left(-\eta N^{-\alpha} / 6\right)$ and deduce that for large normal $r$

for every positive $\beta$.

$$
\begin{aligned}
\sum_{n>(1+n) N} n^{a}\left|a_{n}\right| \rho^{n} & =O\left\{\mu_{0}(\rho) \sum_{n=N}^{\infty} n^{a} t^{n}\right\}=O\left\{N^{Q} \mu_{0}(\rho) t^{N} /(1-t)\right\} \\
& =O\left(\mu_{0}(\rho) N^{-\beta}\right)
\end{aligned}
$$

Suppose next that $n \leq(1-\eta) N$. In this case we write

$$
\rho_{1}=r \exp \left(-N^{-\alpha}\right)
$$

and proceed as above. We deduce that $(1-\alpha) N \leq M \leq N$ and

$$
\begin{aligned}
\frac{\left|a_{n}\right| \rho^{n}}{\mu_{0}(\rho)} & \leq \exp \left\{-\frac{1}{2} \eta N \cdot N^{-\alpha}+N|\log (\rho / r)|\right\} \\
& \leq \exp \left\{-\frac{1}{4} \eta N^{1-\alpha}\right\},
\end{aligned}
$$

if $\alpha$ is given by (3.6) and $N$ is large. Thus

$$
\sum_{n \leq(1-\eta) N} n^{\alpha}\left|a_{n}\right| \rho^{n}=o\left[\mu_{0}(\rho) N^{1+\alpha} \exp \left(-\frac{1}{4} \eta N^{1-\alpha}\right)\right]=o\left[\mu_{0}(\rho) N^{-\beta}\right],
$$

for every positive $\beta$. Thus

for every positive $\beta$.

$$
\sum_{|n-N|>\eta N} n^{q}\left|a_{n}\right| \rho^{n}=o\left\{\mu_{0}(\rho) N^{-\beta}\right\}
$$

Given $\varepsilon>0$, we now choose $\eta$ so small that

$$
b(N+|p|)>(1-\varepsilon) b(N) \text { for }|p| \leq \eta N, \text { and }(1-\eta)^{-\mid \alpha}<1+\varepsilon,
$$

which is always possible under the hypotheses of Theorems $2,3,4$. We also suppose that $r e^{-t} \leq \rho \leq r e^{t}$, where $t=2 k^{-1}$. Then (2.4) yields for $n=N+p,|p|<\eta N$,

$$
\frac{n^{q}\left|a_{n}\right| \rho^{n}}{\mu_{0}(\rho)} \leq(1+\varepsilon) N^{q} e^{t|p|-b p^{2}} \quad \text { where } \quad b=\frac{1}{2}(1-\varepsilon) b(N) .
$$

Thus if $k$ satisfies (3.4) we deduce from this and (3.7) that

$$
\sum_{|n-N| \geq k} n^{\alpha}\left|a_{n}\right| \rho^{n} \leq 2(1+\varepsilon) N^{\alpha} \mu_{0}(\rho)\left\{\sum_{p=k}^{\infty} e^{p t-b p^{2}}+o\left(N^{-\beta}\right)\right\} .
$$


Our hypotheses ensure that, for large $r, N$ is large and $b$ is small, so that $t$ is small compared with $b k$ in view of (3.4). Thus $e^{p t-b p^{2}}$ decreases with increasing $p$, and

$$
\sum_{p=k}^{\infty} e^{p t-b p^{2}} \leq \int_{k-1}^{\infty} e^{t x-b x^{2}} d x
$$

We set $y=x b^{1 / 2}-t /\left(2 b^{1 / 2}\right), y_{0}=(k-1) b^{1 / 2}-t /\left(2 b^{1 / 2}\right)$, and deduce that

and

$$
\int_{k-1}^{\infty} e^{t x-b x^{2}} d x=b^{-1 / 2} e^{\left(t^{2} / 4 b\right)} \int_{y_{0}}^{\infty} e^{-y^{2}} d y
$$

$$
\int_{y_{0}}^{\infty} e^{-y^{2}} d y=\frac{e^{-y_{0}^{2}}}{2 y_{0}}-\int_{y_{0}}^{\infty} \frac{e^{-y^{2}}}{2 y^{2}} d y \sim \frac{e^{-y_{0}^{2}}}{2 y_{0}} .
$$

Thus in view of (3.4) we deduce that

$$
\sum_{p=k}^{\infty} e^{p t-b p^{2}}=O\left\{\frac{e^{-y_{0}^{2}}}{b^{1 / 2} y_{0}}\right\}=O\left\{\frac{\exp \left[\frac{1}{2} \gamma(1-\varepsilon) \log b(N)\right]}{b(N)^{1 / 2}[\log 1 / b(N)]^{1 / 2}}\right\}=O\left\{b^{(1 / 2) \gamma_{1}-(1 / 2)}\right\}
$$

since $\gamma_{1}<\gamma$ and $\varepsilon$ may be chosen as small as we please. Now Lemma 2 follows from (3.8).

We note in particular that

$$
\sum_{|n-N| \geq N}\left|a_{n}\right| r^{n}=o\{\mu(r)\}
$$

if $k>(1+\varepsilon)\left\{b(N)^{-1} \log 1 / b(N)\right\}^{1 / 2}$, for some $\varepsilon>0$, for we can then take $\gamma_{1}=1$ in (3.5).

3.2 Similar estimates for functions of order $(\log r)^{2}$. If $p=2$ in Theorem 4 we obtain a simpler and stronger result. This is

LEMMA 3. If $p=2$ in Theorem 4 and $k$ is a fixed positive integer then

where

$$
\sum_{|n-N| \geq k}\left|a_{n}\right| r^{n} \leq \mu(r) A(\sigma, k),
$$

$$
A(\sigma, k)=2 \sum_{n=k}^{\infty} e^{-(1 / 2) \sigma n^{2}}
$$

We note that if $p=2$ in Theorem 4 , then that result yields for $n=N+k$

$$
\left|a_{n}\right| r^{n} \leq \mu(r) \exp \left(-\frac{\sigma}{2} k^{2}\right) .
$$

outside set $r$ of upper logarithmic density at most $\delta$, provided that $\delta=2 \sigma c e<1$. Thus

$$
\sum_{|n-N| \geq k}\left|a_{n}\right| r^{n} \leq 2 \mu(r) \sum_{t=k}^{\infty} e^{-(1 / 2) \sigma t^{2}}
$$

and this proves Lemma 3 in this case.

3.3 Functions of order $(\log r)^{p}$, when $p<2$. If $p<2$ we use a different technique 
due essentially to Valiron [21, p. 42]. The following result due to Barry [1] will be useful in the sequel, although it is less precise than Lemmas 2 and 3.

LEMMA 4. Suppose that $f(z)$ is an integral function such that

$$
\varlimsup_{r \rightarrow \infty} \frac{\log \log M(r)}{\log \log r}=p<2 .
$$

Then if $0<\eta<2-p$, we have

$$
\sum_{n \neq N}\left|a_{n}\right| r^{n}<\mu(r) \exp \left\{-(\log r)^{\eta}\right\}
$$

outside a set of $r$ of logarithmic density zero.

We choose $\varepsilon>\eta$, and $q>p$, such that $\varepsilon<2-q$. Then it follows from our hypotheses that we have for all sufficiently large $r$

$$
\log \mu(r)<\frac{1}{4}(\log r)^{q} .
$$

Also since $q<2$, we deduce that

so that

$$
N(r) \log r \leq \int_{r}^{r^{2}} N(t) \frac{d t}{t} \leq \log \mu\left(r^{2}\right)<\frac{1}{4}\left(\log r^{2}\right)^{a}<(\log r)^{\alpha},
$$

$$
\log r_{N}>N^{1 /(\alpha-1)}, \quad N>N_{0} .
$$

We now define $k_{N}$ by the equation

and call the set of $r$ for which

$$
\log k_{N}=2\left(\log r_{N}\right)^{\varepsilon}
$$

$$
\frac{r_{N}}{k_{N}} \leq r \leq k_{N} r_{N}
$$

for some $N$ exceptional. We proceed to show that the set of exceptional $r$ has logarithmic density zero. To see this, suppose that

$$
\frac{r_{N}}{k_{N}} \leq r \leq \frac{r_{N+1}}{k_{N+1}}
$$

Then the total logarithmic measure of the exceptional $\rho$, such that $1 \leq \rho \leq r$ is at most

$$
\sum_{n=1}^{N} 2 \log k_{n} \leq 2 N \log k_{N} \leq 4\left(\log r_{N}\right)^{q-1+\varepsilon}=o\left(\log r_{N}\right)=o(\log r)
$$

as required.

Suppose next that $r$ is normal, so that

$$
k_{N} r_{N}<r<\frac{r_{N+1}}{k_{N+1}}
$$


for some $N$, and write $r / r_{N}=c_{1}, r_{N+1} / r=c_{2}$. Then we have for $n<N$

so that

$$
\left|a_{n}\right| r_{N}^{n} \leq a_{N} r_{N}^{N}
$$

so that

$$
\left|a_{n}\right| r^{n} \leq\left|a_{N}\right| r^{N}\left(\frac{r}{r_{N}}\right)^{n-N}=c_{1}^{n-N} \mu(r)
$$

for large $r$. Similarly,

$$
\sum_{n<N}\left|a_{n}\right| r^{n} \leq \mu(r) \sum_{k=1}^{\infty} c_{1}^{-k} \leq \frac{\mu(r)}{c_{1}-1},
$$

$$
\sum_{n>N}\left|a_{n}\right| r^{n} \leq \frac{\mu(r)}{c_{2}-1}
$$

From the fact that $r$ is normal and the definition of $k_{N}$, we deduce that

$$
\frac{1}{c_{1}-1}+\frac{1}{c_{2}-1}=O\left\{\exp -(\log r)^{\varepsilon}\right\}=o\left\{\exp -(\log r)^{\eta}\right\}
$$

and Lemma 4 follows.

4. Maximum modulus and maximum term. It follows from Lemmas 2 to 4 that in the series for $f(z)$ we can neglect those terms whose index is not near $N(r)$. By summing the estimates for the remaining terms we obtain a bound for $M(r)$ in terms of $\mu(r)$. We start by investigating the test functions.

LEMma 5. Suppose that $F(r)$ is any one of the test functions used for the purpose of proving Theorems 2, 3 and the case $p>2$ of Theorem 4. Then if $\mu_{1}(r)$ denotes the maximum term and $N=N_{1}(r)$ the central index of $F(r)$, and if $b(N)$ is defined as in Theorems 2 to 4 , we have $\dagger$

We write

$$
F(r) \sim \mu_{1}(r)\left\{2 \pi / b\left(N_{1}(r)\right)\right\}^{1 / 2}, \quad r \rightarrow \infty .
$$

$$
F(r)=\sum_{0}^{\infty} \alpha_{n} r^{n}
$$

and note that as in Lemma 2 we have

$$
\sum_{|n-N| \geq k} \alpha_{n} r^{n}=o\left\{\mu_{1}(r)\right\}, \quad \text { as } \quad r \rightarrow \infty
$$

if $k$ is given by (3.4) with a suitable constant $\gamma$. In all these cases $k(N)=o(N)$. Thus if $r=\rho_{N}$, we have for all the test functions

$$
\alpha^{\prime}(t)=(1+o(1)) \alpha^{\prime}(N), \text { as } N, t \rightarrow \infty \text {, while }|t-N| \leq k .
$$

Thus in this case, (2.2) can be sharpened to

$$
\frac{\alpha_{n}}{\alpha_{N}} \rho_{N}^{n-N}=\exp \left\{-\frac{1}{2}(n-N)^{2} \alpha^{\prime}(N)(1+o(1))\right\}, \quad|n-N| \leq k .
$$

$\dagger$ For a different class of functions satisfying a similar inequality see [7]. 
Hence, given $\varepsilon>0$, we see that for all sufficiently large $N$

Here

$$
\begin{array}{r}
\sum_{|n-N| \leq k} \alpha_{n}\left(\rho_{N}\right)^{n} \text { lies between } \\
\alpha_{N} \rho_{N}^{N} \sum_{|t| \leq k} \exp \left\{\frac{1}{2} t^{2} \alpha^{\prime}(N)(1 \mp \varepsilon)\right\} .
\end{array}
$$

$$
\alpha^{\prime}(N)=-b(N) .
$$

We write $c=c(N)=-\frac{1}{2} \alpha^{\prime}(N)(1 \mp \varepsilon)$, and note that with our hypotheses $c$ is small if $N$ is large. Thus

$$
\begin{aligned}
\sum_{|t| \leq k} \exp \left(-c t^{2}\right) & =(1+o(1)) \int_{-k}^{k} e^{-c x^{2}} d x=\frac{1+o(1)}{\sqrt{ } c} \int_{-k \sqrt{ } c}^{k \sqrt{ } c} e^{-y^{2}} d y \\
& =\left(1+o(1)(\pi / c)^{1 / 2},\right.
\end{aligned}
$$

since in view of (3.4) $k \sqrt{ } c \rightarrow \infty$, as $N \rightarrow \infty$. Since $\varepsilon$ can be chosen as small as we please, we deduce that

$$
\sum_{|n-N| \leq k} \alpha_{n}\left(\rho_{N}\right)^{n} \sim \alpha_{N} \rho_{N}^{N}\{2 \pi / b(N)\}^{1 / 2} .
$$

Also if $\rho_{N-1} \leq r \leq \rho_{N+1}$, and $|t| \leq k$, we see that

Thus

$$
\left|\log \left\{\left(\frac{r}{\rho_{N}}\right)^{t}\right\}\right| \leq k\left|\log \frac{\rho_{N}}{\rho_{N-1}}\right| \sim k \alpha^{\prime}(N) \rightarrow 0, \text { as } N \rightarrow \infty .
$$

$$
\frac{\sum_{|n-N| \leq k} \alpha_{n} r^{n}}{\alpha_{N} r^{N}} \sim \frac{\sum_{|n-N| \leq k} \alpha_{n} \rho_{N}^{n}}{\alpha_{N} \rho_{N}^{N}} \sim\{2 \pi / b(N)\}^{1 / 2} .
$$

Further if $\rho_{N} \leq r \leq \rho_{N+1}$, the central index of $F(r)$ is either $N$ or $N+1$, and for all our test functions $b(N) \sim b(N+1)$. Thus

$$
\sum_{|n-N| \leq k} \alpha_{n} r^{n} \sim \mu_{1}(r)\{2 \pi / b(N)\}^{1 / 2}
$$

and in view of (4.1) we deduce Lemma 5.

It is sometimes of interest to have an expression for $N$, and hence $b(N)$ in terms of $\mu_{1}(r)$. This can be deduced from (1.8), (2.1) and (4.2). Thus we have in the case of Theorem 2,

$$
\begin{aligned}
b(N) & =-\alpha^{\prime}(N)=\left\{K N \log N \cdots\left(\log _{l+1} N\right)^{1+\delta}\right\}^{-1}, \\
\alpha(N) & =\frac{1}{\delta K}\left(\log _{l+1} N\right)^{-\delta}+c_{1}, \\
\rho_{N} & =c_{2} \exp \left\{-\frac{1}{\delta K}\left(\log _{l+1} N\right)^{-\delta}\right\},
\end{aligned}
$$

where $c_{1}, c_{2} \cdots$ denote positive constants. 
We denote by $e_{l}(x)$ the $l$ times iterated exponential functions. Then for $\rho_{N}<r \leq \rho_{N+1}$, we deduce

$$
N(r) \sim N=e_{l+1}\left\{\frac{c_{3}+o(1)}{\left(c_{2}-r\right)^{1 / \delta}}\right\},
$$

and this in turn yields on integrating by parts

$$
\begin{aligned}
\log \mu(r) & =\int_{r_{0}}^{r} N(t) \frac{d t}{t}+O(1) \\
& \sim c_{4} N\left\{\log N \cdots \log _{l} N \cdots\left(\log _{l+1} N\right)^{(\delta+1)}\right\}^{-1},
\end{aligned}
$$

Thus in this case Lemma 5 yields

$$
F(r) \sim c_{5} \mu(r)(\log \mu(r))^{1 / 2} \log _{2} \mu(r) \cdots \log _{l+1} \mu(r)\left(\log _{l+2} \mu(r)\right)^{1+\delta} .
$$

In the case of Theorem 3, a much simpler analysis yields

$$
\begin{aligned}
\alpha^{\prime}(N) & =-b(N)=-c / N \\
\alpha(t) & =-c \log t+c_{6} \\
\rho_{n} & =c_{7} n^{c}, \\
N(r) & \sim c_{8} r^{1 / c}, \\
\log \mu(r) & \sim c N(r)=c^{2} / b(N) .
\end{aligned}
$$

Thus in this case we obtain from Lemma 5

$$
F(r) \sim \frac{1}{c} \mu_{1}(r)\left\{2 \pi \log \mu_{1}(r)\right\}^{1 / 2} .
$$

Finally in the case of Theorem 4

$$
\begin{aligned}
\alpha^{\prime}(N) & =-b(N)=-\frac{\sigma}{p-1} N^{(2-p) /(p-1)}, \\
\alpha(N) & =-\sigma N^{1 /(p-1)} \\
\rho_{n} & =c_{9} \exp \left\{\sigma N^{1 /(p-1)}\right\}, \\
N(r) & \sim\left(\frac{1}{\sigma} \log r\right)^{p-1} \\
\log \mu(r) & \sim \frac{1}{p} N(r) \log r \sim \frac{\sigma}{p}(N(r))^{p /(p-1)} .
\end{aligned}
$$

Thus in this case Lemma 5 yields

$$
F(r) \sim c_{10} \mu_{1}(r)\left\{\log \mu_{1}(r)\right\}^{(p-2) /(2 p)},
$$

where

$$
c_{10}=(2 \pi)^{1 / 2} p^{(p-2) /(2 p)}(p-1)^{1 / 2} \sigma^{(1-p) / p} .
$$


4.1 The main results. Using (1.12) we can easily deduce from Lemma 5 and (4.3) to (4.5) corresponding inequalities for general functions when $r$ is normal. We have in fact.

THEOREM 5. With the hypotheses of Theorems 2, 3, and 4 when $p>2$, we have, given $\varepsilon>0$,

$$
M(r, f)<(1+\varepsilon) \mu(r)\left\{\frac{2 \pi}{b(N)}\right\}^{1 / 2}
$$

for all sufficiently large normal $r$.

If $p=2$ in Theorem 4, we have with the notation of Lemma 3 for $|z|=r$

$$
\mu(r)(1-A(\sigma, 1)) \leq|f(z)| \leq \mu(r)(1+A(\sigma, 1))
$$

and if $p<2$ and $\varepsilon>0$, we have

$$
|f(z)|=\mu(r)\left\{1+o\left[\exp -(\log r)^{2-p-\varepsilon}\right]\right\} .
$$

Since (4.8) and (4.9) follow immediately from Lemma 3 and 4 respectively it is enough to prove (4.7). Let $F(r)$ be the appropriate test function for which $r$ is normal. Then for normal $r$ we deduce from (1.10) that

$$
\frac{\left|a_{n}\right| r^{n}}{\mu(r)} \leq \frac{\alpha_{n} \rho_{N}^{n}}{\mu_{1}\left(\rho_{N}\right)},
$$

and summing from 0 to $\infty$, we deduce that

$$
\frac{M(r, f)}{\mu(r)} \leq \sum_{0}^{\infty} \frac{\left|a_{n}\right| r^{n}}{\mu(r)} \leq \sum_{0}^{\infty} \frac{\alpha_{n} \rho_{N}^{n}}{\mu_{1}\left(\rho_{N}\right)}=\frac{F\left(\rho_{N}\right)}{\mu_{1}\left(\rho_{N}\right)} \sim\left(\frac{2 \pi}{b(N)}\right)^{1 / 2},
$$

which yields (4.7).

It is not difficult to deduce from (4.5) inequalities in terms of $\mu(r)$ alone. For this purpose we assume that $a_{0}=1$. This result may be achieved by adding a constant to $f(z)$ which evidently does not alter any of our asymptotic relations. Then we may put $n=0$ in (4.10) and deduce that

$$
\frac{1}{\mu(r)} \leq \frac{\alpha_{0}}{\mu_{1}\left(\rho_{N}\right)}
$$

so that

$$
\mu_{1}\left(\rho_{N}\right) \leq \alpha_{0} \mu(r) .
$$

Using the asymptotic relations (4.3) to (4.5) and (4.12), which shows that

$$
\log \mu_{1}\left(\rho_{N}\right) \leq \log \mu(r)+O(1),
$$

we obtain

THEOREM 6. We have for large normal $r$ with the hypotheses of Theorem 2

$$
M(r)<\mu(r)(\log \mu(r))^{1 / 2} \log _{2} \mu(r) \cdots \log _{l} \mu(r)\left[\log _{l+1} \mu(r)\right]^{1+\delta}
$$


With the hypotheses of Theorem 3 we have

$$
M(r)<\frac{1}{c+o(1)} \mu(r)\{2 \pi \log \mu(r)\}^{1 / 2},
$$

and with those of Theorem 4 with $p>2$

$$
M(r)<\left(c_{10}+o(1)\right) \mu(r)(\log \mu(r))^{(p-2) /(2 p)},
$$

where $c_{10}$ is defined by (4.6).

To prove (4.15) for instance, we note that (4.11) and (4.4) yield

$$
\frac{M(r, f)}{\mu(r)} \leq \frac{F\left(\rho_{N}\right)}{\mu_{1}\left(\rho_{N}\right)} \leq \frac{1}{c+o(1)}\left\{2 \pi \log \mu_{1}\left(\rho_{N}\right)\right\}^{1 / 2}
$$

and using (4.13) we deduce (4.15). The other two inequalities follow similarly. It follows from Theorem 3 , that we may take for $c$ any quantity less than $\rho^{-1}$ in (4.15). If $\rho^{\prime}>\rho$, we choose $c$ so that $\rho<c^{-1}<\rho^{\prime}$, and deduce from (4.15) and Theorem 3 that we have

$$
M(r)<\rho^{\prime} \mu(r)\{2 \pi \log \mu(r)\}^{1 / 2}
$$

on a set of lower logarithmic density at least $1-c \rho$. Letting $c^{-1}$ tend to $\rho^{\prime}$, we deduce that the set where (4.17) holds has lower logarithmic density at least $1-\rho / \rho^{\prime}$. On the other hand (4.4) shows that if $\rho^{\prime}<\rho$ the set where (4.17) holds may be bounded.

The inequality (4.14) is also best possible in the sense that $\delta$ cannot be replaced by zero. This can be shown by taking

$$
\alpha(t)=-\log _{l+1}(t)
$$

for large $t$. The corresponding test function is an integral function for which

so that

$$
\alpha^{\prime}(t)=-\left\{t \log t \cdots \log _{l} t\right\}^{-1}=-b(t),
$$

$$
N(r) \sim e_{l}(r), \quad \log \mu(r) \sim N(r)\left\{\log N(r) \cdots \log _{l} N(r)\right\}^{-1},
$$

and so Lemma 5 yields for the corresponding test function $F(r)$

$$
F(r) \sim \mu(r)(2 \pi)^{1 / 2}(\log \mu(r))^{1 / 2} \operatorname{og}_{2} \mu(r) \cdot \cdots \log _{l+1} \mu(r) .
$$

The inequalities (4.14) and (4.15) were proved by Wiman [22] to hold for some arbitrarily large $r$. Valiron [19] showed that (4.14) holds outside a set of finite logarithmic measure. The argument for deducing (4.15) for normal $r$ and hence on a set of positive lower logarithmic density is implicit in the work of Clunle [4].

5. The minimum modulus of functions of small growth. We proceed to prove the following result which goes back to Valiron [21, p. 42].

THEOREM 7. Suppose that $f(z)$ is an integral function, such that

$$
\varlimsup_{r \rightarrow \infty} \frac{\log M(r, f)}{(\log r)^{2}} \leq c<\frac{1}{4 e} .
$$


Then if

$$
m(r, f)=\inf _{|z|=r}|f(z)|,
$$

and $0<4 e c \leq \delta<1$, we have outside a set of upper logarithmic density at most $\delta$

$$
\frac{m(r, f)}{M(r, f)}>K(\delta, c)=\frac{1-2.2 \tau}{1+2.2 \tau}
$$

where $\tau=\exp \{-\delta /(4 c e)\}$.

We define $\sigma$ by $\delta=2 c e \sigma$, so that $\sigma \geq 2$. Then Lemma 3 shows that

$$
\frac{m(r, f)}{M(r, f)} \geq \frac{1-A(\sigma, 1)}{1+A(\sigma, 1)}
$$

for a set of $r$ of lower logarithmic density at least $1-\delta$. Also

$$
A(\sigma, 1)=2 \sum_{k=1}^{\infty} e^{-(1 / 2) \sigma n^{2}}=2 \tau\left(1+\tau^{3}+\tau^{8}+\cdots\right)
$$

where $\tau=e^{-(1 / 2) \sigma}$. In particular, since $\sigma \geq 2$, we see that

Thus

$$
A(\sigma, 1) \leq 2 \tau\left(1+e^{-3}+\frac{e^{-8}}{1-e^{-1}}\right)=2.100 \cdots \tau
$$

as required.

$$
\frac{m(r, f)}{M(r, f)} \geq \frac{1-2.2 \tau}{1+2.2 \tau}
$$

A corresponding result for arbitrary positive $\delta, c$ was proved by Barry [1] with

$$
K(\delta, c)=\exp -\left\{\frac{\pi^{2} c(2-\delta)}{\delta}\right\}
$$

However this beautiful result in which the constant $\pi^{2}$ is best possible is proved by a quite different method and so we omit the proof. Barry [1] also obtains rather sharper estimates than (5.2) for a sequence of $r$ when $c$ is small, but at the cost of density.

We also note the following result of Barry [1] which follows easily from Theorem 7.

THEOREM 8. If (5.1) holds with $c=0$, then

$$
\frac{m(r, f)}{M(r, f)} \rightarrow 1
$$

as $r \rightarrow \infty$ on a set of logarithmic density 1 .

In fact it follows from (5.2) in this case that for every positive $\varepsilon$ the set $E(\varepsilon)$ on which

$$
\frac{m(r, f)}{M(r, f)}>1-\varepsilon
$$


has logarithmic density one. For given $\delta<1$, we may choose $c$ so small that $K(\delta, c)>1-\varepsilon$ in (5.2) so that the complement of $E(\varepsilon)$ has upper logarithmic density at most $\delta$, and so this density must be zero. Since this is true for every $\varepsilon$, Theorem 8 follows. (For details see Barry [1, p. 477].)

If $p<2$ we can prove considerably more. In fact Lemma 4 yields at once

THEOREM 9. If $f(z)$ is an integral function such that

$$
\varlimsup_{r \rightarrow \infty} \frac{\log \log M(r)}{\log \log r}=p<2,
$$

then if $0<\eta<2-p$ we have

$$
\frac{m(r)}{M(r)}>1-\exp \left\{-(\log r)^{\eta}\right\}
$$

outside a set of logarithmic density zero.

6. Behaviour near the local maximum. The Wiman-Valiron Theory can be used to obtain very precise estimates for the behaviour of the function near points on $|z|=r$, where $|f(z)|$ is maximal or nearly maximal, when $r$ is normal. In order to develop this theory we first establish a result on the behaviour of a regular function near a point of maximum modulus. Our result is

Lemma 6. Suppose that $f(z)$ is an integral function and that $\left|z_{0}\right|=r$, $\left|f\left(z_{0}\right)\right|=M(r, f)$. Then we have except possibly for isolated values of $r$

$$
z \frac{d}{d z} \log f(z)=a(r), \quad\left|\left(z \frac{d}{d z}\right)^{2} \log f(z)\right| \leq b(r)
$$

for $z=z_{0}$, where $a(r), b(r)$ are defined by (1.5).

We set $z_{0}=r e^{i \theta}$. Then since $\left|f\left(z_{0}\right)\right|$ is maximal, we deduce that

$$
\frac{\partial}{\partial \theta} \log \left|f\left(r e^{i \theta}\right)\right|=\operatorname{Im} z_{0} \frac{f^{\prime}\left(z_{0}\right)}{f\left(z_{0}\right)}=0 .
$$

Thus $z_{0}$ is one of the finite number of points on $|z|=r$, where $z f^{\prime}(z) \mid f(z)$ is real. In a neighbourhood, $r_{0}-\varepsilon<r<r_{0}+\varepsilon$, of a fixed $r_{0}$, the different values of $\log \left|f\left(r e^{i \theta}\right)\right|$ at the corresponding points $\theta_{v}(r)$ satisfying (6.2) are either identical or distinct, except for isolated values of $r_{0}$. Hence, except for such values of $r_{0}$, for all $r$ sufficiently close to $r_{0}$ the maximum modulus is attained at points $r e^{i \theta(r)}$, where $\theta(r)$ is the solution of (6.2) which coincides with a fixed solution $\theta\left(r_{0}\right)=\theta_{0}$, when $r=r_{0}$, provided that $r_{0} \exp \left(i \theta_{0}\right)$ is a point of maximum modulus.

We now write $z=z_{0} e^{r}$

$$
\log f(z)=\phi(\tau)=\phi(0)+\alpha \tau+(\beta+i \gamma) \tau^{2}+\cdots
$$

where the expansion is valid for small $\tau$. In view of (6.2) it follows that

$$
\alpha=z_{0} \frac{f^{\prime}\left(z_{0}\right)}{f\left(z_{0}\right)}
$$


is purely real. Next we choose $\tau=\sigma+i \theta$, where $\theta=\theta\left(r_{0} e^{\sigma}\right)-\theta_{0}$, so that $z$ is a point of maximum modulus. We assume that the maximum modulus curve does not touch $|z|=r$ at $z_{0}$, which is true except for isolated values of $r$. Then it follows from (6.3) that

so that

$$
\log \frac{M\left(r_{0} e^{\sigma}\right)}{M\left(r_{0}\right)}=\log \left|\frac{f(z)}{f\left(z_{0}\right)}\right|=\alpha \sigma+O\left(\sigma^{2}\right),
$$

$$
a\left(r_{0}\right)=\frac{d}{d \sigma} \log M\left(r_{0} e^{\sigma}\right)=\alpha=z_{0} \frac{f^{\prime}\left(z_{0}\right)}{f\left(z_{0}\right)},
$$

which is the first conclusion of (6.1). To obtain the second inequality we assume that $\beta \neq 0$, which is true except for isolated values of $r$ and choose $\theta=-\gamma \sigma / \beta$, $\tau=\sigma+i \theta$. Since $\beta=-(\partial / \partial \theta)^{2} \log \left|f\left(r_{0} e^{i \theta}\right)\right| \geq 0$ in any case. we may thus assume $\beta>0$. Taking real parts in (6.3) we deduce

$$
\begin{aligned}
\log M\left(r_{0} e^{\sigma}\right) & \geq \log \left|f\left(z_{0} e^{\tau}\right)\right|=\log \left|f\left(z_{0}\right)\right|+\alpha \sigma+\beta\left(\sigma^{2}-\theta^{2}\right)-2 \gamma \sigma \theta+O\left(\sigma^{3}\right) \\
& =\log M\left(r_{0}\right)+\alpha \sigma+\frac{\beta^{2}+\gamma^{2}}{\beta} \sigma^{2}+O\left(\sigma^{3}\right) .
\end{aligned}
$$

We deduce that

$$
\begin{aligned}
b\left(r_{0}\right) & =\left(\frac{d}{d \sigma}\right)^{2} \log M\left(r_{0} e^{\sigma}\right) \geq \frac{2\left(\beta^{2}+\gamma^{2}\right)}{\beta} \geq 2\left(\beta^{2}+\gamma^{2}\right)^{1 / 2} \\
& =\left|\left(z \frac{d}{d z}\right)^{2} \log f(z)\right|
\end{aligned}
$$

at $z=z_{0}$. This proves the second inequality in Lemma 6 and completes the proof of that Lemma.

We need some more subsidiary results.

Lemma 7. Suppose that $P(z)$ is a polynomial of degree $m$, and $|P(z)| \leq M$, for $|z| \leq r$. Then if $R \geq r$, we have

$$
\left|P^{\prime}(z)\right| \leq \frac{e M m R^{m-1}}{r^{m}}, \quad|z| \leq R .
$$

In fact it follows from a classical result of Bernstein [2, see also 13 p. 221] that the result holds without the factor $e$. However this does not greatly affect our results and we can give a simple proof of Lemma 7.

We note first that $P(z) / z^{m}$ remains regular at $\infty$ and so we can apply the maximum principle to this function in $|z|>r$ and deduce that

$$
|P(z)| \leq M\left(\frac{|z|}{r}\right)^{m}, \quad|z|>r .
$$


Now we apply Cauchy's inequality and deduce that for $|z| \leq R, h=R / m$

$$
\left|P^{\prime}(z)\right| \leq \frac{1}{h} \max _{|\zeta-z| \leq h}|P(\zeta)| \leq \frac{M}{h r^{m}}(R+h)^{m}=\frac{M m R^{m-1}}{r^{m}}\left(1+\frac{1}{m}\right)^{m} .
$$

This yields the required result. We deduce

Lemma 8. With the hypotheses of Lemma 7 if $\left|z_{0}\right| \leq r,\left|P\left(z_{0}\right)\right| \geq \eta M$, where $0<\eta \leq 1$, then we have

for $\left|z-z_{0}\right|<\eta r \mid(8 m)$.

$$
\frac{1}{2}\left|P\left(z_{0}\right)\right|<|P(z)|<\frac{3}{2}\left|P\left(z_{0}\right)\right|
$$

We deduce from Lemma 7 that for $\left|z-z_{0}\right| \leq \eta r /(8 m)$

$$
\left|P^{\prime}(z)\right| \leq \frac{e M m}{r^{m}} r^{m-1}\left(1+\frac{1}{8 m}\right)^{m-1}<\frac{4 m M}{r} .
$$

Thus in this range

$$
\left|P(z)-P\left(z_{0}\right)\right|=\left|\int_{z_{0}}^{z} P^{\prime}(\xi) d \xi\right| \leq \frac{4 M m}{r}\left|z-z_{0}\right| \leq \frac{1}{2} M \eta \leq \frac{1}{2}\left|P\left(z_{0}\right)\right| .
$$

6.1 Statement and proof of the main results. We can now state and prove our first result on local behaviour $\dagger$

THEOREM 10. Suppose that $f(z)$ is an integral function and that $r$ is normal in the sense of one of the Theorems $2,3,4$, provided that $p>2$ in the latter case. In this case we also set $\gamma=10(p-1) /(p-2)$, while in the other cases we set $\gamma=10$. We write $N$ for $N(r)$, suppose that $\left|z_{0}\right|=r$ and

$$
\left|f\left(z_{0}\right)\right| \geq \eta M(r, f) \text { where } N^{-3} \leq \eta \leq 1,
$$

and define $k$ by (3.4). Then if

$$
z=z_{0} e^{\tau}, \text { and }|\tau| \leq \eta /(30 k)
$$

we have

$$
\log \frac{f(z)}{f\left(z_{0}\right)}=\left(N+\phi_{1}\right) \tau+\phi_{2} \tau^{2}+\delta(\tau)
$$

where

$$
\left|\phi_{j}\right| \leq \frac{4}{\pi}(18 k / \eta)^{j}, \quad j=1,2, \quad \text { and } \quad|\delta(\tau)| \leq 4(18 k|\tau| / \eta)^{3} .
$$

We apply Lemma 2 with $\gamma$ defined as in Theorem 10. Then we have for $\gamma_{1}<\gamma$ and $|z|=\rho$, where $|\rho-r|<r \mid k$

$$
f(z)=\sum_{N-k}^{N+k} a_{n} z^{n}+o\left\{\mu_{0}(\rho) b(N)^{(1 / 2) \gamma_{1}-(1 / 2)}\right\} .
$$

$\dagger$ Similar results which are however not quite correct in the dependence of $\tau$ upon $\eta$ were obtained by Clunie [4, Theorem 5 and 5, Theorem 9]. 
In the case of Theorems 2 and 3, we have

$$
b(N)=O\left(N^{-1}\right),
$$

so that taking $\gamma_{1}=9$, we deduce

$$
f(z)=\sum_{N-k}^{N+k} a_{n} z^{n}+o\left\{\mu_{0}(\rho) N^{-4}\right\}=\sum_{N-k}^{N+k} a_{n} z^{n}+o\left\{M(\rho, f) N(r)^{-4}\right\} .
$$

In the situation of Theorem 4, we have

$$
b(N)=O\left\{N^{(2-p) /(p-1)}\right\}
$$

so taking $\gamma_{1}=9(p-1) /(p-2)$, we again deduce (6.8), so that (6.8) holds in all cases.

Thus if $|f(z)| \geq M(\rho, f) N(r)^{-4}$, we have

$$
(1+o(1)) f(z)=z^{N-k} P(z),
$$

where $P(z)$ is a polynomial of degree at most $2 k$. To this polynomial we apply Lemma 7. We deduce from (6.8) that we have for $|z|=r$

$$
\left|z^{N-k} P(z)\right| \leq(1+o(1)) M(r, f),
$$

so that if $\varepsilon$ is a fixed positive number and

$$
M_{0}=(1+\varepsilon) r^{k-N} M(r, f),
$$

we have, when $r$ is sufficiently large

$$
|P(z)| \leq M_{0}, \quad|z|=r .
$$

Also (6.4) and (6.8) show that

$$
f\left(z_{0}\right)=z^{N-k} P\left(z_{0}\right)+o\left\{M(r, f) N(r)^{-4}\right\}=z^{N-k} P\left(z_{0}\right)+o\left\{f\left(z_{0}\right)\right\}
$$

so that for large $r$

Thus

$$
r^{N-k}\left|P\left(z_{0}\right)\right| \geq(1-\varepsilon)\left|f\left(z_{0}\right)\right| \geq(1-\varepsilon) \eta M(r, f) .
$$

$$
\left|P\left(z_{0}\right)\right| \geq \frac{1-\varepsilon}{1+\varepsilon} \eta M_{0} .
$$

Hence we deduce from Lemma 8 that

$$
\frac{1}{2}\left|P\left(z_{0}\right)\right|<|P(z)|<\frac{3}{2}\left|P\left(z_{0}\right)\right| \text { for }\left|z-z_{0}\right|<\frac{1-\varepsilon}{1+\varepsilon} \frac{\eta r}{16 k} .
$$

Also for this range of $z$ we have (6.8) so that

$$
\begin{aligned}
\frac{f(z)}{z^{N}} & =\frac{P(z)}{z^{k}}+o\left\{\frac{\mu_{0}(\rho)}{\rho^{N}} N(r)^{-4}\right\}=\frac{P(z)}{z^{k}}+o\left\{a_{N} N^{-4}\right\} \\
& =\frac{P(z)}{z^{k}}+o\left\{\frac{M(r, f)}{r^{N}} N^{-4}\right\}=\frac{P(z)}{z^{k}}+o\left\{\frac{M_{0}}{r^{k} N^{4}}\right\}=\frac{(1+o(1) P(z))}{z^{k}},
\end{aligned}
$$


since for large $k$

$$
\frac{14}{15} \leq\left(1-\frac{1}{16 k}\right)^{k} \leq\left(\frac{\rho}{r}\right)^{k}=\left|\frac{z}{z_{0}}\right|^{k} \leq\left(1+\frac{1}{16 k}\right)^{k} \leq \frac{16}{15} .
$$

Using this and (6.10) we deduce finally that

$$
|f(z)|=\left|f\left(z_{0}\right)\right|\left|\frac{z}{z_{0}}\right|^{N}(1+H),
$$

where

We now consider

$$
|H|<\frac{3}{5}, \text { for }\left|z-z_{0}\right|<\frac{\eta r}{17 k} .
$$

in the disk

$$
\phi(\tau)=\log f\left(z_{0} e^{\tau}\right)-N \tau-\log f\left(z_{0}\right)
$$

$$
|\tau| \leq \tau_{0}=\frac{\eta}{18 k} .
$$

Then if $\phi(\tau)=u+i v$, and $r$ is large, so that $k$ is large, we deduce from (6.11) that

$$
|u| \leq \log \frac{5}{2}<1 \quad|\tau| \leq \tau_{0} .
$$

Also $\phi(0)=0$. Hence if

$$
\phi(\tau)=\sum_{1}^{\infty} \phi_{n} \tau^{n}, \quad|\tau|<\tau_{0},
$$

we deduce from subordination [see e.g. 13, p. 221] that

$$
\left|\phi_{n}\right| \leq \frac{4}{\pi} \tau_{0}^{-n},
$$

so that

$$
\left|\sum_{n=3}^{\infty} \phi_{n} \tau^{n}\right| \leq \frac{4}{\pi} \frac{|\tau|^{3}}{\tau_{0}^{2}\left(\tau_{0}-|\tau|\right)} .
$$

In particular we deduce that

where

$$
\phi(\tau)=\phi_{1} \tau+\phi_{2} \tau^{2}+\delta(\tau),
$$

$$
|\delta(\tau)|<\frac{10}{\pi}\left(\frac{|\tau|}{\tau_{0}}\right)^{3} \text { for }|\tau| \leq \frac{3}{5} \tau_{0}=\frac{\eta}{30 k} .
$$

On combining (6.12) and (6.13) we have Theorem 10.

In view of Lemma 6 we also deduce

THEOREM 11. If $\left|f\left(z_{0}\right)\right|=M(r, f)$, so that $\eta=1$ in Theorem 10 , we have $N(r)+\phi_{1}=a(r),\left|\phi_{2}\right| \leq \frac{1}{2} b(r)$ in Theorem 10 where $a(r), b(r)$ are defined as in (1.5). 
Theorem 10 asserts in particular that $f(z) \neq 0$ for $\left|\log \left(z / z_{0}\right)\right|<\eta /(30 k)$. If $f(z)$ has finite order $\rho$ it follows from Theorem 3 that we may take $b(N)=c_{1} / N$, and so $k=c_{2}(N(\log N))^{1 / 2}$ where $c_{1}, c_{2}$ are constants depending on $\rho$. Also the smaller we take $c_{1}$ and so the larger we take $c_{2}$, the smaller does the density of the exceptional set of $r$ become.

6.2. Behaviour of derivatives. The Wiman-Valiron theory also permits us to give good estimates for the derivatives of $f(z)$, when $|z|$ is normal. We proceed to prove $\dagger$

THEOREM 12. Suppose that $f(z)$ and $r$ satisfy the hypotheses of Theorem 10, that $\gamma$ and $k$ are defined as in that Theorem and that

$$
r\left(1-\frac{1}{40 k}\right)<\rho<r\left(1+\frac{1}{40 k}\right) .
$$

Then if $q$ is a fixed nonnegative integer we have for $|z|=\rho$

$$
\left(\frac{z}{N}\right)^{q} f^{(q)}(z)=f(z)+O\left(\frac{k}{N}\right) M(\rho, f) .
$$

In particular if $\log (\rho / r)=o\left(k^{-1}\right)$, then

$$
M\left(\rho, f^{(q)}\right)=\left(\frac{N(r)}{\rho}\right)^{q}\left[1+O\left(\frac{k}{N(r)}\right)\right] M(\rho, f) \sim\left(\frac{N(r)}{r}\right)^{q}\left(\frac{\rho}{r}\right)^{N} M(r, f) .
$$

We write

$$
f(z)=\sum_{N-k}^{N+k} a_{n} z^{n}+\phi(z)=z^{N-k} P(z)+\phi(z) .
$$

Thus we have by Lemma 2

$$
\begin{aligned}
\rho^{q}\left|\phi^{(a)}(z)\right| & =\left|\sum_{|n-N| \geq k} a_{n} n(n-1) \cdots(n-q+1) z^{n}\right| \\
& \leq \sum_{|n-N|>k} n^{q}\left|a_{n}\right| \rho^{n}=o\left\{\mu_{0}(\rho) N^{q} b(N)^{(1 / 2) \gamma_{1}-(1 / 2)}\right\} .
\end{aligned}
$$

for any $\gamma_{1}<\gamma$. We again choose $\gamma_{1}=\cdot 9 \gamma$ and deduce just as in the proof of Theorem 10 , that

so that

$$
b(N)^{1 / 2 \gamma_{1}-1 / 2}=O\left(N^{-4}\right),
$$

$$
\rho^{\alpha}\left|\phi^{(\alpha)}(z)\right|=o\left\{\mu_{0}(\rho) N^{q-4}\right\} .
$$

Again repeated application of Lemma 7 shows that if $P(z)$ is the polynomial occurring in (6.16) then for any fixed $v \geq 0$, and $|z|=\rho$, we have

$$
\left|P^{(v)}(z)\right|=O(k / r)^{v} M_{0},
$$

$\dagger$ A result of this type is (not quite correctly) stated and proved by Clunie [5, Theorem 8]. 
where $M_{0}$ is the quantity in (6.9). Thus

$$
\begin{aligned}
\left(\frac{d}{d z}\right)^{q} z^{N-k} P(z) & =\sum_{v=0}^{q}\left(\begin{array}{l}
q \\
v
\end{array}\right) \frac{(N-k) !}{(N-k-q+v) !} z^{N-k-q+v} P^{(v)}(z) \\
& =\frac{(N-k) !}{(N-k-q) !} z^{N-k-q}\left\{P(z)+O(k / N) M_{0}\right\} \\
& =\frac{(N-k) !}{(N-k-q) !} z^{-q}\left\{f(z)+O\left(\mu_{0}(\rho) N^{-4}\right)+O\left(\rho^{N-k} M_{0} k / N\right)\right\}
\end{aligned}
$$

in view of (6.16) and (6.17). Now

Also

$$
\mu_{0}(\rho) N^{-4} \leq M(\rho, f) N^{-4}=O\left(\frac{k}{N}\right) M(\rho, f) .
$$

$$
\rho^{N-k} M_{0}=(1+\varepsilon)\left(\frac{\rho}{r}\right)^{N-k} M(r, f)=O\left(\frac{\rho}{r}\right)^{N} M(r, f) .
$$

Thus combining (6.16), (6.17) and (6.18) we deduce that

$$
z^{q} f^{(q)}(z)=\frac{(N-k) !}{(N-k-q) !}\left\{f(z)+O\left(\frac{k}{N}\right) M(\rho, f)+O\left(\frac{\rho}{r}\right)^{N}\left(\frac{k}{N}\right) M(r, f)\right\} .
$$

Next we chose $z_{0}$ so that $\left|f\left(z_{0}\right)\right|=M(r, f)$ and take $\eta=1, \tau=\log (\rho / r)$. Then (6.6) gives

so that

$$
\begin{aligned}
\log \left|f\left(\frac{\rho}{r} z_{0}\right)\right| & =\log \left|f\left(z_{0}\right)\right|+N \tau+O(1), \\
\tau & =O(1) / k
\end{aligned}
$$

$$
\log M(\rho, f) \geq \log M(r, f)+N \log \frac{\rho}{r}+O(1)
$$

and hence

$$
\left(\frac{\rho}{r}\right)^{N} M(r, f)=O\{M(\rho, f)\}
$$

Thus (6.19) yields

$$
z^{q} f^{(q)}(z)=\frac{(N-k) !}{(N-k-q) !}\left\{f(z)+O\left(\frac{k}{N}\right) M(\rho, f)\right\}=N^{q}\left\{f(z)+O\left(\frac{k}{N}\right) M(\rho, f)\right\},
$$

which is (6.14).

We chose $z$ in (6.14) in turn so as to make $|f(z)|$ and $\left|f^{(q)}(z)\right|$ maximal and deduce that

and

$$
M\left(\rho, f^{(q)}\right) \geq\left\{1+O\left(\frac{k}{N}\right)\right\}\left(\frac{N}{\rho}\right)^{q} M(\rho, f)
$$

$$
M\left(\rho, f^{(\alpha)}\right) \leq\left\{1+O\left(\frac{k}{N}\right)\right\}\left(\frac{N}{\rho}\right)^{q} M(\rho, f),
$$


so that

$$
M\left(\rho, f^{(q)}\right)=\left(\frac{N}{\rho}\right)^{q}\left\{1+O\left(\frac{k}{N}\right)\right\} M(\rho, f) .
$$

To complete the proof of $(6.15)$ it remains to show that

$$
\log M(\rho, f)=\log M(r, f)+N \log \frac{\rho}{r}+o(1) .
$$

To see this we note that (6.16) and (6.17) yield for our range of $\rho$

$$
\log M(\rho, f)=(N-k) \log \rho+\log M(\rho, P)+o(1) .
$$

On the other hand it follows from Lemma 7, that

$$
M(\rho, P)=M(r, P)\{1+O(k(\rho-r) / r)\} \sim M(r, P)
$$

if $k \log (\rho / r)=o(1)$, and now the second inequality of (6.15) also follows and the proof of Theorem 12 is complete.

6.3. A generalization of Picard's Theorem. We can use the preceding results to prove a result which generalizes Picard's Theorem. This is

THEOREM 13.† Suppose that $f(z)$ is a transcendental integral function. Then $f(z)$ assumes every value with at most one exception infinitely often and, if such an exceptional value exists, all the derivatives $f^{(a)}(z)$ assume every value except possibly zero infinitely often.

We note that if $f(z)=e^{z}+a$, then $f(z) \neq a, f^{(q)}(z) \neq 0$ for any $q \geq 1$. We suppose that the equation $f(z)=0$ has only a finite number of roots and deduce that for any $q \geq 0$, the equation $f^{(\alpha)}(z)=1$ has infinitely many roots. This is equivalent to the stated result since if the equations $f(z)=a, f^{(q)}(z)=b$ have only a finite number of roots, where $b \neq a$, if $q=0$, and $b \neq 0$, if $q>0$, we may consider $(f(z)-a) /(b-a)$ or $(f(z)-a) / b$ instead of $f(z)$.

Suppose then that $f(z)$ has only a finite number of zeros. Then

$$
f(z)=P(z) e^{g(z)},
$$

where $P(z)$ is a polynomial and $g(z)$ an integral function. Suppose first that $g(z)$ is a polynomial. Then it is elementary to show that $f(z)$ assumes all values except zero infinitely often [see e.g. 17, p. 279]. Since $f^{(p)}(z)$ takes a similar form, we also deduce that $f^{(p)}(z)$ assumes all values except zero infinitely often in this case.

Suppose next that $g(z)$ is a transcendental integral function. Then we apply Theorem 2 and its consequences with $b(N)=N^{-1}(\log N)^{-3}$, so that we may take in (3.4) and subsequently

$$
k=10\left[N^{1 / 2}(\log N)^{2}\right]
$$
[3].

$\dagger$ The case $q=1$ appears to be due to Pólya-Saxer $[15$, p. 210] and the general case to Bureau 
where $N=N(r)$ is the central index. We note that

$$
f^{\prime}(z)=\left(P^{\prime}+P g^{\prime}\right) e^{g}, f^{\prime \prime}=\left(P g^{\prime 2}+2 P^{\prime} g^{\prime}+P g^{\prime \prime}+P^{\prime \prime}\right) e^{g},
$$

and prove by induction that

$$
f^{(a)}(z)=Q(z) e^{g},
$$

where $Q(z)$ is a polynomial of degree $q$ in $g^{\prime}(z)$ and its derivatives, with coefficients that are polynomials in $z$, and the leading term of $Q(z)$ is $P g^{\prime q}$.

It now follows from Theorems 10 and 12 that if $r$ is normal, $z_{0}$ is so chosen that $\left|g\left(z_{0}\right)\right|=M(r, g),\left|z_{0}\right|=r$ and if $\left|\log z / z_{0}\right|=0\left(N^{-1}\right)$ where $N(r)$ is the central index of $g(z)$, we have

Thus

$$
g^{(v)}(z) \sim\left(\frac{N}{z}\right)^{v} g(z)=O\left\{\left(\frac{N}{r}\right)^{v} M(r, g)\right\}
$$

On the other hand

$$
P(z) g^{\prime}(z)^{q} \sim P(z)\left(\frac{N}{z_{0}}\right)^{q} g(z)^{\alpha} .
$$

$$
N(r)=r \frac{d}{d r} \log \mu(r)<\{\log \mu(r)\}^{2} \leq(\log M(r))^{2}
$$

outside a set of finite logarithmic measure. In fact if $E$ is the set in $\left[r_{0}, \infty\right]$ where (6.20) is false, we have in view of (1.7)

$$
\int_{E} \frac{d r}{r} \leq \int_{E} \frac{N(r) d r}{r \log \mu(r)^{2}} \leq \int_{r_{0}}^{\infty} \frac{N(r) d r}{r \log \mu(r)^{2}}=\frac{1}{\log \mu\left(r_{0}\right)}<\infty .
$$

Thus we assume that $r$ is outside this exceptional set and then

$$
Q(z) \sim P(z)\left(\frac{N}{z_{0}}\right)^{q} g(z)^{q},
$$

since the other terms in the polynomial for $Q(z)$ only contribute terms of the order $M(r, g)^{q-1+\varepsilon}$. We have shown that

$$
f^{(\alpha)}(z) \sim P(z)\left(\frac{N g(z)}{z_{0}}\right)^{q} e^{g(z)}, \text { for }\left|\log \left(\frac{z}{z_{0}}\right)\right|=|\tau| \leq 10 / N
$$

say. We deduce that in the same disk

$$
h(z)=\log f^{(\alpha)}(z)=g(z)+q \log \frac{N}{z_{0}}+q \log g(z)+\log P(z)+o(1) \sim g(z)
$$

in view of $(6,20)$. Hence in this range, if we set $H(z)=\log h(z)$, we deduce that with a suitable choice of $H\left(z_{0}\right)$ we have

$$
H(z)=\log g(z)+o(1)=H\left(z_{0}\right)+N \tau+o(1)
$$

in view of Theorem 10 . 
We now apply Rouchés theorem and deduce that if $\left|w-H\left(z_{0}\right)\right|<5$, then the equation $H(z)=w$ has a root for $|N \tau|<10$, i.e. $\left|\log \left(z / z_{0}\right)\right|<10 N^{-1}$. We may choose $w$ of the form $w=\log (2 \pi l)+\left(2 m+\frac{1}{2}\right) \pi i$, for some integers $l$ and $m_{0}$. In fact let $H\left(z_{0}\right)=U_{0}+i V_{0}$, so that $U_{0}$ is large and positive. Then we may choose $l$ so that

$$
\left|\log (2 \pi l)-U_{0}\right|<1 \text {, and } m \text { so that }\left|\left(2 m+\frac{1}{2}\right) \pi-V_{0}\right| \leq \pi,
$$

and this gives $\left|w-H\left(z_{0}\right)\right|<5$ as required. Thus there exists $z_{1}$, such that $H\left(z_{1}\right)=w$, $h\left(z_{1}\right)=e^{w}=2 \pi i l, f^{(p)}\left(z_{1}\right)=\exp \left(h\left(z_{1}\right)\right)=1$. Since there are infinitely many such points $z_{1}$, Theorem 13 is proved. It is worth stating that more precise results applying also to meromorphic functions can be obtained by means of Nevanlinna Theory [8, Theorem 3.5] but the present approach is probably more elementary. It also shows that for functions of infinite order the roots are located near the points of maximum modulus. This is false in general for functions of finite order as $f(z)=e^{z}$ shows.

7. Two Counterexamples: The sigma function. To test the sharpness of the results obtained we consider two examples.

EXAMPLE 1. Let $\sigma(z)$ be the sigma function with zeros at all the lattice points $z_{m, n}=m+n i$. Then

$$
\mathfrak{p}(z)=-\left(\frac{d}{d z}\right)^{2} \log \sigma(z)=z^{-2}+\sum_{m, n}^{\prime}\left\{\left(\frac{1}{z-z_{m, n}}\right)^{2}-\frac{1}{z_{m, n}^{2}}\right\}
$$

and the dash denotes the fact that the term for $m=n=0$ is to be omitted. Then $\mathfrak{p}(z)$ is clearly doubly periodic with periods 1 and $i$.

Also integrating we deduce that

satisfies

$$
\zeta(z)=\frac{\sigma^{\prime}(z)}{\sigma(z)}
$$

$$
\zeta(z+1)-\zeta(z)=A, \quad \zeta(z+i)-\zeta(z)=-A i,
$$

where $A$ is a real constant. The second equation follows from the first, since by inspection

satisfies $\zeta(i z)=-i \zeta(z)$.

$$
\zeta(z)=\frac{1}{z}+\Sigma^{\prime}\left\{\frac{1}{z-z_{m, n}}+\frac{z}{z_{m, n}^{2}}\right\}
$$

Integrating again we deduce that

Also

$$
\log \frac{\sigma(z+1)}{\sigma(z)}=A z+B, \quad \log \frac{\sigma(z+i)}{\sigma(z)}=-A i z+C .
$$

$$
\sigma(z)=z \exp \prod_{m, n}^{\prime}\left(1-\frac{z}{z_{m, n}}\right) \exp \left\{\frac{z}{z_{m, n}}+\frac{z^{2}}{2 z_{m, n}^{2}}\right\}
$$

is clearly an odd function. So setting $z=-\frac{1}{2},-\frac{1}{2} i$, we deduce that 
so that

$$
e^{B-(A / 2)}=-1, \quad e^{C-(A / 2)}=-1,
$$

$$
\sigma(z+1)=-e^{A(z+1 / 2)} \sigma(z), \quad \sigma(z+i)=-e^{-A i(z+i / 2)} \sigma(z) .
$$

We now deduce

LEMMA 9. If $z_{2}=z_{1}+m+n i$, where $m, n$ are integers then

$$
\left|\sigma\left(z_{2}\right)\right| e^{-1 / 2 A\left|z_{2}\right|^{2}}=\left|\sigma\left(z_{1}\right)\right| e^{-1 / 2 A\left|z_{1}\right|^{2}} .
$$

Write $z_{1}=x+i y, z_{2}=x+1+i y$. Then

$$
\left|\frac{\sigma\left(z_{2}\right)}{\sigma\left(z_{1}\right)}\right|=\left|e^{A\left(z_{1}+1 / 2\right)}\right|=e^{A(x+1 / 2)}=e^{1 / 2 A\left(\left|z_{2}\right|^{2}-\left|z_{1}\right|^{2}\right)} .
$$

Similarly if $z_{2}=z_{1}+i$

$$
\left|\frac{\sigma\left(z_{2}\right)}{\sigma\left(z_{1}\right)}\right|=\left|e^{-A i(z+i / 2)}\right|=e^{A(y+1 / 2)}=e^{1 / 2 A\left(\left|z_{2}\right|^{2}-\left|z_{1}\right|^{2}\right)}
$$

Thus we see that $|\sigma(z)| \exp \left(-\frac{1}{2} A|z|^{2}\right)$ is invariant, if $z$ is increased by a primitive period and so by any period.

If $z=z_{1}$ is an arbitrary point in the plane we can always choose $z_{2}=x_{2}+i y_{2}$, so that $\left|x_{2}\right| \leq \frac{1}{2},\left|y_{2}\right| \leq \frac{1}{2}$. Then $\left|z_{2}\right|$ is the distance from $z$ to the nearest zero of $\sigma(z)$, and if we denote this distance by $d$, we see that there exist positive constants $A_{1}$, $A_{2}$ such that

Thus

$$
A_{1} d \leq\left|\sigma\left(z_{2}\right)\right| e^{-A\left(\left.z_{2}\right|^{2}\right.} \leq A_{2} d
$$

$$
A_{1} d e^{1 / 2 A|z|^{2}} \leq|\sigma(z)| \leq A_{2} d e^{1 / 2 A|z|^{2}},
$$

where $d$ is the distance from $z$ to the nearest zero.

It is clear that $A>0$, since otherwise $\sigma(z)$ would be constant by Liouville's Theorem. $\dagger$ Thus $\sigma(z)$ has order 2 mean type, and in fact

$$
\begin{aligned}
\log M(r) & =\frac{A}{2} r^{2}+O(1), \\
\log \mu(r) & \sim \frac{A}{2} r^{2}, \\
N(r) & \sim A r^{2} .
\end{aligned}
$$

For all values of $z_{0}$ on $\left|z_{0}\right|=r$ there is a zero in $\left|z-z_{0}\right| \leq 1 / \sqrt{ } 2$, whereas Theorem 10 asserts that there is no such zero in

$$
\left|z-z_{0}\right| \leq \operatorname{cr}\{N(r) \log N(r)\}^{-1 / 2}
$$

Thus the index $\frac{1}{2}$ in (3.4) and in the corresponding estimate for $\tau$ in (6.5) cannot be replaced by any smaller quantity.

$\dagger$ In fact an application of Jensen's formula shows at once that $A=\pi / 2$. 
Theorem 10 also shows that if $\left|f\left(z_{0}\right)\right|>\eta \exp \left(\frac{1}{2} A r^{2}\right)$ then $f(z)$ has an asymptotic formula and so $f(z) \neq 0$ for $\left|z-z_{0}\right|<c \eta /(\log r)^{1 / 2}$, where $c$ is a constant. This gives the right result apart from the factor $(\log r)^{-1 / 2}$. Clunie $[4,5]$ had stated Theorems of the type of Theorem 10 but with $|\tau| \leq A / k$ instead of $|\tau| \leq \eta /(30 k)$, where $A$ is a constant. The above examples show that no Theorem of this kind can be true. However Clunie's argument correctly yields Theorem 10 for functions of finite order.

To see the size that $\eta$ may take in Theorem 10 for this example we investigate the minimum modulus of $\sigma(z)$, or what is the same thing, the distance of an arbitrary circle $|z|=r \geq 1$ to the nearest lattice point. Given $r$, let $x$ be the fractional part of $r^{2}$. Then if $z=m+$ in is any zero $\left.\left|r^{2}-\right| z\right|^{2} \mid \geq \min (x, 1-x)$, so that for most $r,(r-|z|) \geq c / r$ for all zeros, where $c$ is a positive constant and so $\eta \geq c^{1} N(r)^{-1 / 2}$ in Theorem 10. On the other hand if we choose for $m$ the largest integer, such that $m^{2} \leq r^{2}$, and then for $n$ the largest integer such that $m^{2}+n^{2} \leq r^{2}$, we see that

$$
r^{2}-m^{2} \leq 2 m+1=O(r), \quad r^{2}-m^{2}-n^{2} \leq 2 n+1=O\left(r^{1 / 2}\right),
$$

so that if $z=m+i n,(r-|z|)=O\left(r^{-1 / 2}\right)$. Thus we can always find a point on $|z|=r$, where

$$
f(z)=O\left\{M(r, f) r^{-1 / 2}\right\}=O\left\{M(r, f) N(r)^{-1 / 4}\right\} .
$$

Thus $\eta$ can take any value between $c N(r)^{-1 / 4}$ and 1 , for any positive $r$, and for any such $\eta$, the estimate for the disk in which an asymptotic expansion holds, is sharp, apart from a possible factor $(\log r)^{-1 / 2}$.

7.1. How large must $k$ and $\eta$ be? Another example. It is natural to ask whether the factor $\{-\log b(N)\}^{1 / 2}$ which occurs in the definition of $k$ in (3.4) and so in (6.5) is really necessary. We proceed to give an example to show that this is the case.

For this purpose we set

$$
\alpha^{\prime}(t)=-\frac{2}{t}, \quad t \geq 1, \quad \alpha^{\prime}(t)=-2, \quad t \leq 1
$$

in the construction of section 2 , and then, with a suitable choice of $K$ we have

$$
\alpha(t)=-2(\log t+1), \quad t \geq 1, \quad \rho_{n}=e^{2} n^{2}, \quad \alpha_{n}=n^{-2 n}, \quad n \geq 1,
$$

in (2.1), and $b(N)=2 / N$. We now set

$$
\phi_{1}(z)=\sum^{\prime} \alpha_{n} z^{n}, \quad \phi_{2}(z)=\sum_{0}^{\infty} \alpha_{n} z^{n}
$$

where the sum $\Sigma^{\prime}$ is taken over certain indices which will now be described. We define a sequence $\lambda_{v}$ inductively by

$$
\lambda_{v+1}=\lambda_{v}+\left[\frac{1}{2}\left(\lambda_{v} \log \lambda_{v}\right)^{1 / 2}\right], \quad v \geq 1, \quad \lambda_{1}=4,
$$


where $[x]$ denotes the integral part of $x$. We then sum $\Sigma^{\prime}$ in (7.3) over all those powers $n$ for which, for some $v \geq 1$, we have

$$
\lambda_{v} \leq n \leq \lambda_{v}+\frac{1}{4} \lambda_{v}^{1 / 2}, \quad \text { or } n=\lambda_{v} \mp\left[\varepsilon\left(\lambda_{v} \log \lambda_{v}\right)^{1 / 2}\right],
$$

where $\varepsilon$ is a positive constant, which we take to be small. We write

$$
d_{v}=\left[\varepsilon\left(\lambda_{v} \log \lambda_{v}\right)^{1 / 2}\right], \quad \lambda_{v}^{\prime}=\lambda_{v}+d_{v}, \quad \lambda_{v}^{\prime \prime}=\lambda_{v+1}-d_{v+1} .
$$

Then it follows from the analysis of section 1.2 that, for

$$
e^{2} \lambda_{v}^{\prime 2} \leq r \leq e^{2} \lambda_{v}^{\prime \prime 2}
$$

the central index $N_{1}(r)$ of $\phi_{1}(z)$ is either $\lambda_{v}^{\prime}$ or $\lambda_{v}^{\prime \prime}$. Also, if $\mu_{1}(r), \mu_{2}(r)$ are the maximum terms of $\phi_{1}(z), \phi_{2}(z)$, then $\mu_{1}(r) \leq \mu_{2}(r)$. Further if $r$ lies in the range (7.5) then the central index $N_{2}(r)$ of $\phi_{2}(z)$ satisfies

$$
\lambda_{v}^{\prime} \leq N_{2}(r) \leq \lambda_{v}^{\prime \prime} .
$$

Now the analysis of section 2 shows that if

$$
\lambda_{v} \leq n \leq \lambda_{v}+\frac{1}{4} \lambda_{v}^{1 / 2}
$$

and $r$ lies in the range (7.5) so that $N_{2}(r)$ satisfies (7.6) we have

$$
\frac{\alpha_{n} r^{n}}{\mu_{2}(r)}=\exp \left\{-(1+o(1)) \frac{(n-N)^{2}}{N}\right\},
$$

where $N=N_{2}(r)$. Thus if we sum over the range (7.7) and $r$ is sufficiently large, we deduce that

where $k=N-\lambda_{v}$. Thus

$$
\frac{\sum \alpha_{n} r^{n}}{\mu_{2}(r)}>\frac{\lambda_{v}^{1 / 2}}{4} \exp \left\{-\frac{6}{5} \frac{k^{2}}{N}\right\}
$$

$$
\begin{aligned}
\frac{\sum \alpha_{n} r^{n}}{\mu(r)} & >\frac{\lambda_{v}^{1 / 2}}{4} \exp \left\{\frac{-5\left(\lambda_{v+1}-\lambda_{v}\right)^{2}}{4 \lambda_{v}}\right\} \\
& >\frac{\lambda_{v}^{1 / 2}}{4} \exp \left\{\frac{-5 \log \lambda_{v}}{16}\right\}>\lambda_{v}^{1 / 6},
\end{aligned}
$$

when $\nu$ is large. Thus for $r$ in the range (7.5), we see that the terms in the range (7.7) are much larger than $\mu_{2}(r)$ and à fortiori than $\mu_{1}(r)$.

A similar analysis holds for the range (7.7) with $v$ replaced by $v+1$. The sum of these terms is also much larger than $\mu_{2}(r)$. On the other hand the remaining terms of $\phi_{1}(z)$ can be shown to be small compared with these so that for $r$ in the range (7.5) we have

$$
(1+o(1)) \phi_{1}(r)=\Sigma_{1} \alpha_{n} r^{n}+\Sigma_{2} \alpha_{n} r^{n}
$$

where in $\Sigma_{1}$ we sum over the $n$ satisfying (7.7), while in $\sum_{2}$ we sum over the $n$ satisfying (7.7), with $(v+1)$ instead of $v$. The indices in $\sum_{1}$ and $\sum_{2}$ satisfy respectively

$$
n \leq N(r)-\frac{\varepsilon}{2} N(r)^{1 / 2} \log N(r)^{1 / 2}, \quad n \geq N(r)+\frac{\varepsilon}{2} N(r)^{1 / 2}\left(\log N(r)^{1 / 2}\right) .
$$


Thus for $r$ satisfying (7.5) we cannot neglect both these ranges of terms in computing $\phi_{1}(r)$. Also the logarithmic density of all the ranges (7.5) is

$$
\lim _{v \rightarrow \infty} \frac{\log \left(e^{2} \lambda_{v}^{\prime \prime 2}\right)-\log \left(e^{2} \lambda_{v}^{\prime 2}\right)}{\log \left(e^{2} \lambda_{v+1}^{2}\right)-\log \left(e^{2} \lambda_{v}^{2}\right)}=\lim _{v \rightarrow \infty} \frac{(1-4 \varepsilon) \lambda_{v}^{3 / 2} \log \lambda_{v}}{\lambda_{v}^{3 / 2} \log \lambda_{v}}=1-4 \varepsilon .
$$

This can be chosen as near 1 as we please, by choosing $\varepsilon$ small enough. Thus Lemma 2 fails if $\gamma_{1}=1$, and $\gamma$ is sufficiently small.

Clearly $\phi_{c}(z)$ always attains its maximum modulus on the positive real axis. It is not difficult to see that if $\Sigma_{1}, \Sigma_{2}$ are defined as in (7.8) then in the range (7.5) we have for $z=r e^{i \tau}$

if $\tau=o\left(\lambda_{n}^{-1 / 2}\right)$, while

$$
\Sigma_{1} \alpha_{n} z^{n}=e^{i \lambda_{n} \tau}\left(1+o(1) \Sigma_{1} \alpha_{n} r^{n}\right.
$$

$$
\Sigma_{2} \alpha_{n} z^{n}=e^{i \lambda_{n+1} \tau}(1+o(1)) \Sigma_{2} \alpha_{n} r^{n} .
$$

Thus provided that one of these sets of terms dominates the other a formula of the type given in (6.6) holds, but with $\lambda_{n}$ or $\lambda_{n+1}$ instead of $N(r)$. The difference is significant unless $\left(\lambda_{n+1}-\lambda_{n}\right) \tau \rightarrow 0$, i.e. unless $\tau=o\{N \log N\}^{-1 / 2}$, which is the order of magnitude for $k^{-1}$ implied by (3.4) in this case. This limitation for the validity of (6.6) is therefore sharp, when $\eta=1$ in Theorem 10.

8. Maximum and minimum of the real part. We finish the paper by investigating the functions $A(r)$ and $B(r)$ of (1.4) in their relation to the maximum modulus $M(r)$. The following classical result is an immediate consequence of Theorem 10 .

THEOREM 14 [Wiman, 23]. If $f(z)$ is a transcendental integral function and $A(r)$, $B(r), M(r)$ are defined by (1.4) and (1.2) respectively then we have

$$
-A(r) \sim B(r) \sim M(r)
$$

as $r \rightarrow \infty$ outside an exceptional set of finite logarithmic measures.

We apply Theorem 10 with $\eta=1$ taking $b(N)=\left\{N(\log N)^{2}\right\}^{-1}, k=N^{1 / 2}(\log N)^{3 / 2}$. Then (6.6) yields for $|\tau| \leq 2 \pi N^{-1}, z=z_{0} e^{\tau}$

$$
\log \frac{f(z)}{f\left(z_{0}\right)}=N \tau+\frac{O(\log N)^{3 / 2}}{N^{1 / 2}} .
$$

Suppose that $z_{0}$ is chosen so that $f\left(z_{0}\right)=M \exp (i \lambda)$, where $M=M(r, f)$ and $-\pi \leq \lambda<\pi$. We choose $\tau=-i \lambda / N$ and obtain for $z=z_{0} \exp (i \tau)$

so that

$$
\log f(z)=\log M+o(1)
$$

and hence

$$
f(z)=u(z)+i v(z)=(1+o(1)) M
$$

$$
B(r) \geq(1+o(1)) M
$$

Similarly by choosing $\tau=i(\pi-\lambda) / N$, we obtain for $z=z_{0} e^{\tau}$

$$
\log f(z)=(-1+o(1)) M
$$


so that

$$
A(r) \leq(-1+o(1)) M .
$$

Since clearly $|A(r)| \leq M, B(r) \leq M$, we obtain (8.1). Also in view of Theorem 2 we see that the exceptional set of $r$ has finite logarithmic measure. This proves Theorem 14.

If we are prepared to admit a larger exceptional set we can obtain a much more precise result. This is

THEOREM 15.† Suppose that $f(z)$ is a transcendental integral function and set

$$
\varlimsup_{r \rightarrow \infty} \frac{\log \log M(r)}{\log \log r}=p .
$$

Then given $\varepsilon>0$, we have on a set of $r$ of positive upper logarithmic density

$$
B(r)>M(r)\left(1-\frac{\pi^{2}(\alpha(p)+\varepsilon)}{2 \log M(r)}\right), \quad-A(r)>M(r)\left(1-\frac{\pi^{2}(\alpha(p)+\varepsilon)}{2 \log M(r)}\right)
$$

where $\alpha(p)=0$ if $p<2, \alpha(p)=(p-1) / p$, if $2 \leq p<\infty, \alpha(p)=1$ if $p=\infty$.

In the opposite direction we have

THEOREM 16. $\dagger$ For any $p$, such that $2 \leq p \leq \infty$, there exists $f(z)$ satisfying (8.2) and such that we have as $r \rightarrow \infty$

$$
B(r)=M(r), \quad-A(r)=M(r)\left(1-\frac{\pi^{2}(\alpha(p)+o(1))}{2 \log M(r)}\right)
$$

Clearly by considering $-f(z)$ instead of $f(z)$ we obtain a corresponding example with $-A(r)$ and $B(r)$ interchanged.

8.1. Proof of Theorem 15: preliminaries. We apply the preceding theory with

$$
b(N)^{-1}=N(\log N)^{2}, \quad k=N^{1 / 2}(\log N)^{3 / 2} .
$$

Then the set of exceptional $r$ has finite logarithmic measure and if $r$ is normal, and if $\left|z_{0}\right|=r, f\left(z_{0}\right)=M \exp (i \lambda), M=M(r, f)$, Theorem 11 yields

$$
\log f\left(z_{0} e^{i \theta}\right)=\log M+i(\lambda+\theta a(r))-\frac{1}{2} \phi_{2} \theta^{2}+O\left\{|\theta|^{3} N(r)^{3 / 2+\delta}\right\}
$$

for every positive $\delta$ and $|\theta|=o\left(k^{-1}\right)$. By comparing Theorems 10 and 11 we also deduce that

$$
N(r) \sim a(r) \text { as } r \rightarrow \infty \text { for normal values. }
$$

We now choose $\theta=h / a(r)$, where $|h| \leq \pi$. Then the above asymptotic formula certainly yields

$$
\log f\left(z_{0} e^{i \theta}\right)=\log M+i(\lambda+h)-\phi_{2} \frac{h^{2}}{a(r)^{2}}+O\left(a(r)^{-3 / 2+\delta}\right) .
$$

$\uparrow$ Not previously published. 
We may suppose that $|\lambda| \leq \pi$. Then if we choose $h=-\lambda$, we obtain

$$
\log f\left(z_{0} e^{i \theta}\right)=\log M-\phi_{2} \frac{h^{2}}{a(r)^{2}}+O\left(a(r)^{-3 / 2+\delta}\right) .
$$

Thus if $f\left(z_{0} e^{i \theta}\right)=u+i v$

so that

$$
u+i v=M \exp \left\{-\phi_{2} \frac{h^{2}}{a(r)^{2}}+O\left(a(r)^{-3 / 2+\delta}\right)\right\},
$$

$$
\begin{aligned}
B(r) \geq u(z) & \geq M\left\{1-\frac{(1+o(1)) \pi^{2}\left|\phi_{2}\right|}{a(r)^{2}}+O\left(a(r)^{-3 / 2+\delta}\right)\right\} \\
& \geq M\left\{1-\frac{(1+o(1)) \pi^{2} b(r)}{2 a(r)^{2}}+O\left(a(r)^{-3 / 2+\delta}\right)\right\}
\end{aligned}
$$

in view of Theorem 11. By choosing $h=\pi-\lambda$, if $\lambda>0$ and $h=-\pi-\lambda$ if $\lambda<0$, we obtain a similar inequality for $-A(r)$.

To complete the proof we proceed to estimate the quantities occurring in (8.4). We have first

LEMMA 9. Let $f(x)$ be a positive increasing and convex function of $x$ for $x \geq x_{0}$ and suppose that

$$
\underset{x \rightarrow \infty}{\lim } \frac{\log f(x)}{\log x} \leq p \leq \varlimsup_{x \rightarrow \infty} \frac{\log f(x)}{\log x}, \text { where } p>1 \text {. }
$$

Let $\alpha(p)=(p-1) / p$ if $p<\infty ; \alpha(p)=1$, if $p=\infty$. Suppose that $a, K$ are constants such that $K>1$, and $a<\alpha(p)$. Then if $E$ is the set of all $x$ such that $\dagger$

$$
\frac{f(x) f^{\prime \prime}(x)}{f^{\prime}(x)^{2}} \leq K \alpha(p), \quad \text { and } f^{\prime}(x)>f(x)^{a},
$$

we have $\overline{\operatorname{dens}} E \geq(K-1) / K$.

Suppose first that $p$ is finite. Then we choose $p_{1}, p_{2}$ such that $p_{1}<p<p_{2}$ and $\alpha\left(p_{1}\right)>a$. Then by hypothesis we can choose $x_{1}$ as large as we please such that

$$
f\left(x_{1}\right)>x_{1}^{p_{1}} \text {. }
$$

Let $C$ be a large positive constant and suppose that

$$
\frac{f^{\prime}(x)}{f(x)}>\frac{p_{2}}{x}, \text { for } C x_{1}<x<x_{2} .
$$

Then we deduce that

$$
f\left(x_{2}\right) \geq f\left(C x_{1}\right)\left(\frac{x_{2}}{C x_{1}}\right)^{p_{2}} \geq C^{-p_{2}} x_{1}^{p_{1}}\left(\frac{x_{2}}{x_{1}}\right)^{p_{2}} .
$$

$\dagger$ Since $f(x)$ is convex, $f^{\prime \prime}(x)$ exists almost everywhere. We define $f^{\prime}(x)$ to be the right derivative of $f(x)$, so that $f^{\prime}(x)$ is increasing for all $x \geq x_{0}$ and continuous outside a countable set. 
In view of (8.5) this inequality must be false for some arbitrary large $x_{2}$. Thus we choose $x_{2}$ maximal subject to (8.7), and deduce that

$$
\frac{f^{\prime}\left(x_{2}\right)}{f\left(x_{2}\right)} \leq \frac{p_{2}}{x_{2}}, \quad x_{2} \geq C x_{1}
$$

and also that we have

$$
f(x) \geq C^{-p_{2} x^{p_{1}},}, \quad x_{1} \leq x \leq x_{2} .
$$

We next note that $f^{\prime}(x)$ increases so that

$$
f(x)=f\left(x_{0}\right)+\int_{x_{0}}^{x} f^{\prime}(t) d t \leq f\left(x_{0}\right)+\left(x-x_{0}\right) f^{\prime}(x) \leq 2 x f^{\prime}(x)
$$

when $x$ is large enough. Thus we deduce from (8.10) that

$$
f^{\prime}(x) \geq \frac{f(x)}{2 x} \geq f(x) \frac{1}{2}\left(\frac{C^{-p_{2}}}{f(x)}\right)^{1 / p_{1}}>f(x)^{a}, \quad x_{1} \leq x \leq x_{2}
$$

provided that $x_{1}$ is large enough. Also (8.9) holds.

If $p$ is infinite, we choose $p_{1}$ so that $\alpha\left(p_{1}\right)>a$. We may then still choose $x_{1}$ as large as we please to satisfy (8.6) and in this case we set $x_{2}=C x_{1}$ and deduce that

$$
f(x) \geq x_{1}^{p_{1}} \geq C^{-p_{1}} x^{p_{1}}, \quad x_{1} \leq x \leq x_{2},
$$

and this in turn leads to

$$
f^{\prime}(x)>f(x)^{a}, \quad x_{1} \leq x \leq x_{2} .
$$

Thus we deduce that in all cases we can find $x_{1}$ as large as we please and $x_{2} \geq C x_{1}$ such that (8.11) holds and in addition (8.9) holds if $p$ is finite with any $p_{2}>p$.

We now note that if

$$
\phi(x)=x-\frac{f(x)}{f^{\prime}(x)}, \quad \text { then } \phi^{\prime}(x)=\frac{f(x) f^{\prime \prime}(x)}{f^{\prime}(x)^{2}}
$$

almost everywhere. Also for $x \geq x_{0}, h>0$, we have, since $f^{\prime}(t)$ increases

$$
\frac{f(x+h)}{f^{\prime}(x+h)}=\frac{f(x)+\int_{x}^{x+h} f^{\prime}(t) d t}{f^{\prime}(x+h)} \leq \frac{f(x)+h f^{\prime}(x+h)}{f^{\prime}(x+h)} \leq \frac{f(x)}{f^{\prime}(x)}+h .
$$

Thus $\phi(x)$ is non-decreasing. Suppose now that $E_{1}$ is the subset of $\left[x_{1}, x_{2}\right]$ in $E$ and let $E_{2}$ be the rest of the interval $\left[x_{1}, x_{2}\right]$. Then we have almost everywhere in $E_{2}$

$$
\phi^{\prime}(x)=\frac{f(x) f^{\prime \prime}(x)}{f^{\prime}(x)^{2}}>K \alpha(p)
$$

since (8.11) holds in $E_{2}$. Since $\phi(x)$ increases with $x$ we deduce that

$$
\phi\left(x_{2}\right)-\phi\left(x_{0}\right) \geq \int_{x_{0}}^{x_{2}} \phi^{\prime}(t) d t \geq \int_{E_{2}} \phi^{\prime}(t) d t \geq K \alpha(p) m\left(E_{2}\right),
$$


so that

If $p=\infty$, this gives

$$
m\left(E_{2}\right) \leq \frac{\phi\left(x_{2}\right)-\phi\left(x_{0}\right)}{K \alpha(p)}
$$

$$
m\left(E_{2}\right) \leq \frac{x_{2}}{K}+O(1)
$$

If $p<\infty$, we may apply (8.9) and deduce that

$$
\phi\left(x_{2}\right) \leq\left(1-\frac{1}{p_{2}}\right) x_{2}=\alpha\left(p_{2}\right) x_{2}
$$

Thus in this case we deduce that

Thus we have in this case

$$
m\left(E_{2}\right) \leq \frac{\alpha\left(p_{2}\right)}{K \alpha(p)} x_{2}+O(1) .
$$

$$
m\left(E_{1}\right)=\left(x_{2}-x_{1}\right)-m\left(E_{2}\right) \geq x_{2}\left(1-\frac{\alpha\left(p_{2}\right)}{K \alpha(p)}-\frac{1}{C}+\frac{O(1)}{x_{2}}\right) .
$$

This is true for some arbitrary large $x_{2}$ and hence

$$
\overline{\mathrm{dens}} E \geq 1-\frac{\alpha\left(p_{2}\right)}{K \alpha(p)}-\frac{1}{C} \text {. }
$$

Since we may choose $C$ as large as we please and $p_{2}$ as near $p$ as we please we deduce that $\overline{\operatorname{dens}} E \geq 1-K^{-1}$. The same conclusion follows from (8.12) if $p=\infty$, and this completes the proof of Lemma 9.

8.2 Completion of the proof of Theorem 15. We suppose first that $p>3$ in (8.2). In this case we set $r=e^{x}$, and apply Lemma 9 with

$$
f(x)=\log M\left(e^{x}\right), \quad f^{\prime}(x)=a\left(e^{x}\right), f^{\prime \prime}(x)=b\left(e^{x}\right),
$$

choose $a$ so that $\frac{2}{3}<a<1-1 / p$ and let $E$ be the set of $r$ for which

$$
\frac{b(r) \log M(r)}{a(r)^{2}} \leq K \alpha(p) \text { and } a(r)>(\log M(r))^{a} .
$$

Then, by Lemma $9, E$ has upper logarithmic density at least $1-K^{-1}$. Also for normal $r$ on $E$ we have, in view of (8.4),

$$
B(r) \geq M(r)\left(1-\frac{K \pi^{2} \alpha(p)}{2 \log M(r)}+\frac{o(1)}{\log M(r)}\right)
$$

provided that $\delta$ is chosen so small that $((3 / 2)-\delta) a>1$. Given $K_{1}>K$, we deduce that

$$
B(r) \geq M(r)\left(1-\frac{K_{1} \pi^{2} \alpha(p)}{\log M(r)}\right)
$$


on a set of upper logarithmic density at least $1-1 / K$. Since we may choose $K$ as near $K_{1}$ as we please, Theorem 15 follows.

Suppose now that $2 \leq p \leq 3$. Then we may apply Theorems 10,11 as before but now we can use the better estimate for $k$ and hence $\tau$, which follows from Theorem 4 , with any $p_{1}>3$ and $\delta=0$. In particular we may choose $b(N) \geq N^{-1 / 2-\delta}$, for any $\delta>0$, and so $k<N^{1 / 4+\delta}$ for any $\delta>0$. When we apply this to Theorem 10 we see that we obtain the improved error term

$$
\delta(\tau)=O\left(\tau^{3} N^{3 / 4+3 \delta}\right)=O\left\{N^{-9 / 4+3 \delta}\right\}=O\left(a(r)^{-9 / 4+3 \delta}\right)
$$

in (8.4). We can now apply Lemma 9 again, but with $a$ so chosen that $a<\frac{1}{2}$, $9 a / 4>1$, and we again obtain (8.13). Since the set of non-normal $r$ has zero logarithmic density the conclusion again holds on a set of upper logarithmic density at least $(K-1) / K$.

Finally suppose that $1 \leq p<2$. In this case we apply Lemma 4 . We suppose that $r$ is a quantity satisfying the conditions of that Lemma with some $\eta<2-p$, and deduce that

$$
M(r)<\mu(r)\left\{1+\exp \left(-(\log r)^{\eta}\right)\right\} .
$$

We also choose $\theta$ so that if $z=r e^{i \theta}$ then $a_{N} z^{N}$ is real and positive. Then

$$
B(r) \geq a_{N} z^{N}-\sum_{n \neq N}\left|a_{n} z^{n}\right| \geq \mu(r)\left\{1-\exp \left(-(\log r)^{\eta}\right)\right\} .
$$

Thus if $r$ is large

$$
M(r)-B(r)<2 \mu(r) \exp \left(-(\log r)^{\eta}\right) \leq 2 M(r) \exp \left\{-\log M(r)^{\eta^{\prime} / \mathfrak{p}}\right\},
$$

for any $\eta^{\prime}<\eta$, and this result is stronger than (8.13). This completes the proof of Theorem 15.

8.3. Proof of Theorem 16. We proceed to construct our examples. Suppose first that $p=\infty$. In this case we set $f(z)=e^{z}$. We have for $z=r e^{i \theta}$

$$
u=\exp (r \cos \theta) \cos \{r \sin \theta\} .
$$

Choose $\theta=\delta / r$, where $|\delta| \leq \pi$. Then we have uniformly for $\theta$ in this range as $r \rightarrow \infty$

$$
u=e^{r}\left\{1-\frac{\delta^{2}}{2 r}+O\left(r^{-3}\right)\right\} \cos \left\{\delta+O\left(r^{-2}\right)\right\} .
$$

To make $-u$ as large as possible $|\delta|$ must be near to $\pi$. Thus we have throughout this range

$$
-u \leq e^{r}\left(1-\frac{\pi^{2}+o(1)}{2 r}\right)
$$

and for $\pi / r \leq|\theta| \leq \pi$, we have evidently

$$
-u \leq \exp (r \cos \theta) \leq \exp (r \cos \pi / r) \text {. }
$$


Thus (8.14) holds uniformly as $r \rightarrow \infty$ for $|\theta| \leq \pi$, and we deduce that

$$
-A(r) \leq e^{r}\left(1-\frac{\pi^{2}+o(1)}{2 r}\right)=M(r)\left\{1-\frac{\pi^{2}+o(1)}{2 \log M(r)}\right\} .
$$

By taking $\theta=\pi / r$, we deduce that

$$
-A(r) \geq \exp (r \cos \pi / r)=e^{r}\left(1-\frac{\pi^{2}+o(1)}{2 r}\right) .
$$

Evidently $B(r)=M(r)=e^{r}$, so that Theorem 16 is proved in this case.

Suppose next that $2 \leq p<\infty$. In this case we set

where

$$
f_{\mathfrak{p}}(z)=\prod_{n=1}^{\infty}\left(1+\frac{z}{\rho_{n}}\right)
$$

$$
\rho_{n}=\exp \left\{n^{1 /(p-1)}\right\}, \quad p>2 ; \quad \rho_{n}=\exp \{n / \log n\}, \quad p=2 .
$$

It is evident that $\left|f\left(r e^{i \theta}\right)\right|$ attains its maximum for given $r$ when $\theta=0$ and decreases steadily with increasing $|\theta|$ for $0 \leq|\theta| \leq \pi$. To obtain an estimate for $f\left(r e^{i \theta}\right)$ when $\theta$ is small, we proceed as follows. We write

$$
b_{p}(z)=\left(z \frac{d}{d z}\right)^{2} \log f_{p}(z)=\sum_{n=1}^{\infty} \frac{\rho_{n} z}{\left(z+\rho_{n}\right)^{2}}, \quad a_{p}(z)=z \frac{d}{d z} \log f(z) .
$$

Suppose first that $z=\rho_{N}$, and set $n=N+k$. Then for $p>2,|k| \leq \frac{1}{2} N$

$$
\frac{\rho_{n}}{\rho_{N}}=\exp \left\{(N+k)^{1 /(p-1)}-N^{1 /(p-1)}\right\}=\exp \left\{\frac{k N^{(2-p) /(p-1)}}{p-1}+O\left(k^{2} N^{(3-2 p) /(p-1))}\right\} .\right.
$$

We set

and deduce that for $k=o(N)$

$$
c_{N}=\frac{N^{(2-p) /(p-1)}}{p-1}
$$

This leads to

$$
\frac{\rho_{n}}{\rho_{N}}=\exp \left\{-k c_{N}(1+o(1))\right\}
$$

$$
b_{p}\left(\rho_{N}\right) \sim \sum_{k=-\infty}^{+\infty}\left(e^{1 / 2 k c_{N}}+e^{-1 / 2 k c_{N}}\right)^{-2} \sim \int_{-\infty}^{\infty} \frac{d x}{\left(e^{1 / 2 c_{N} x}+e^{-1 / 2 c_{N} x}\right)^{2}}=\frac{1}{c_{N}} .
$$

Since $\rho_{n} / \rho_{n+1} \rightarrow 1$, as $n \rightarrow \infty$, we deduce that for $\rho_{n}<\rho<\rho_{n+1}$

$$
b_{p}(\rho) \sim b_{p}\left(\rho_{n}\right) \sim(p-1) n^{(p-2) /(p-1)} \sim(p-1)(\log \rho)^{p-2},
$$

and integrating twice with respect to $\log r$ yields

$$
a_{p}(\rho) \sim(\log \rho)^{p-1}, \quad \log f_{p}(\rho) \sim \frac{(\log \rho)^{p}}{p} .
$$


It is not difficult to see that the above asymptotic expressions for $b_{p}(\rho), a_{p}(\rho)$ and $\log f_{p}(\rho)$ remain valid when we set $\rho=z$, and allow $z$ to be complex, provided that $|\arg z|<\pi-\delta$ for some positive $\delta$. For instance a straightforward estimate shows that in this range

$$
\left|b_{p}(z)\right|=O(\log |z|)^{p-2},
$$

and now we can use Montel's theorem to show that

$$
\frac{b_{p}(z)}{(\log z)^{p-2}} \rightarrow p-1, \quad \text { as }|z| \rightarrow \infty
$$

uniformly for $|\arg z|<\pi-\delta$, for any fixed $\delta$. From this we can deduce that for $|\tau|<\pi / 2$, say and $\rho$ real and positive, we have

$$
\log f_{p}\left(\rho e^{i \tau}\right)=\log f_{p}(\rho)+i \tau a_{p}(\rho)-\frac{1}{2} \tau^{2} b_{p}(\rho)+O|\tau|^{3}\left|b_{p}(\rho)\right| .
$$

If we set $f_{p}\left(\rho e^{i \tau}\right)=u+i v$, this gives for $\tau$ real and $|\tau|\left|a_{p}(\rho)\right| \leq \pi$

$$
u=f_{p}(\rho)\left(1-\frac{1}{2} \tau^{2} b_{p}(\rho)+O|\tau|^{3} b_{p}(\rho)\right) \cos \left(\tau a_{p}(\rho)+O|\tau|^{3} b_{p}(\rho)\right),
$$

and we deduce that for $u$ to have its minimum value in this range we must have $|\tau| a_{p}(\rho) \sim \pi$. Thus for $|\tau| \leq \pi / a_{p}(\rho)$

$$
u \geq-f_{p}(\rho)\left[1-\frac{\pi^{2} b_{p}(\rho)}{2 a_{p}(\rho)^{2}}(1+o(1))\right]=-f_{p}(\rho)\left(1-\frac{\left(\pi^{2}+o(1)\right)(p-1)}{2 p \log f_{p}(\rho)}\right) ;
$$

for $\pi / a_{p}(\rho) \leq \theta \leq \pi$, we have

Thus we deduce that

$$
\begin{aligned}
\left|u\left(\rho e^{i \theta}\right)\right| & \leq\left|f_{p}\left(\rho e^{i \theta}\right)\right| \leq \mid f_{p}\left\{\rho\left(\exp i \pi / a_{p}(\rho)\right\} \mid\right. \\
& =f_{p}(\rho)\left(1-\frac{\left(\pi^{2}+o(1)\right)(p-1)}{2 p \log f_{p}(\rho)}\right) .
\end{aligned}
$$

$$
\begin{aligned}
A(r, u) & =-f_{p}\left\{\rho \exp \left(i \pi / a_{p}(\rho)\right)\right\}+o\left\{f_{p}(\rho) / \log f_{p}(\rho)\right\} \\
& =-f_{p}(\rho)\left\{1-\frac{\left(\pi^{2}+o(1)\right) \alpha(p)}{2 \log f_{p}(\rho)}\right\} .
\end{aligned}
$$

When $p=2$ the above argument has to be modified slightly, since an asymptotic formula for $b(\rho)$ only holds if $\rho_{n+1} / \rho_{n} \rightarrow 1$. We have

$$
\rho_{n}=\exp (n / \log n)
$$

from (8.15) and then the argument goes through essentially as before, with

so that

$$
c_{N}=\frac{1}{\log N}, \quad b_{2}\left(\rho_{N}\right) \sim \frac{1}{c_{N}}=\log N,
$$

$$
\begin{gathered}
b_{2}(\rho) \sim \log \log \rho, \quad a_{2}(\rho) \sim \log \rho \log \log \rho, \\
\log f_{2}(\rho) \sim \frac{1}{2}(\log \rho)^{2} \log \log \rho .
\end{gathered}
$$


We again deduce that if $A_{2}(r)$ is the minimum of the real part of $f_{2}(z)$, then

$$
\begin{aligned}
-A_{2}(r) & =f_{2}(r)\left(1-\frac{\pi^{2}\left(1+o(1) b_{2}(r)\right.}{2 a_{2}(r)^{2}}\right) \\
& =f_{2}(r)\left(1-\frac{\pi^{2}+o(1)}{4 \log f_{2}(r)}\right)
\end{aligned}
$$

so that Theorem 16 holds when $p=2$. This completes our proof.

9. Conclusion. The foregoing results represent some of the main achievements of Wiman-Valiron theory. Its development in the present form is due to many people, but the principal credit after the original authors is probably due to Clunie $[4,5]$, who sharpened the form of the error term and also introduced the notion of density in connection with the theory, as it occurs for instance in Theorem 3, and to Kövari [11], who developed the very nice form of comparison sequences in section 2 . This has made the basic theory much less formidable than it used to be.

A number of developments have been left out. For instance Theorem 5 lends almost immediately to a relation between the maximum modulus and characteristic of functions with Picard values [cf. 9]. Another subject to which the theory has been successfully applied is that of power series with gaps [see e.g. 6, 10, 11, 16]. However it seems to me that many of the latter applications, which deal with terms well away from the central index, can be obtained by means of more elementary methods, such as Cauchy's inequality combined with growth Lemmas similar to Lemma 1.

The theory as it stands seems dependent on upper growth, since in the development of section 1.2 the comparison sequences $\alpha_{n}$ have to be larger than $\left|a_{n}\right|$ for all $n$. Recently this disadvantage of the theory has to some extent been overcome by Sons [16] who has obtained results for functions of finite lower order but without conclusions about density. Some extensions of this general nature would seem to be desirable in order to replace upper logarithmic density by lower logarithmic density or alternatively to replace the upper limit by the lower limit in the statement of Theorem 15.

The Wiman-Valiron theory can be extended to functions in the unit disk (see e.g. [12]) but Kövari feels that the probabilistic technique of Rosenbloom [14] is generally more successful here.

I am most grateful to the referee for carefully reading through the whole of this paper and correcting some errors.

\section{REFERENCES}

1. P. D. Barry, The minimum modulus of small integral and subharmonic functions, Proc. London Math. Soc. (3) 12 (1962), 445-495.

2. S. Bernstein, Sur l'ordre de la meilleure approximation des fonctions continues par des polynômes de degré donné, Mém de l'Acad. Royale de Belgique (2) 4 (1912), 1-104. 
3. F. Bureau, Sur quelques propriétés des fonctions uniformes au voisinage d'un point singulier essentiel isolé, C. R. de l'acad. des sciences (Paris) 192 (1931), 1350-1352.

4. J. Clunie, The determination of an integral function of finite order by its Taylor series, J. London Math. Soc. 28 (1953), 58-66.

5. J. Clunie, On the determination of an integral function from its Taylor series, J. London Math. Soc. 30 (1955), 32-42.

6. W. H. J. Fuchs, Proof of a conjecture of G. Pólya concerning gap series, Illinois J. Math. 7 (1963), 661-667.

7. W. K. Hayman, A generalisation of Stirling's formula, J. Reine Angew. Math. 196 (1956), $67-95$.

8. W. K. Hayman, Meromorphic functions (Oxford, 1964).

9. W. K. Hayman and F. M. Stewart, Real inequalities with applications to function theory, Proc. Cambridge Philos. Soc. 50 (1954), 250-260.

10. T. Kövari, On theorems of G. Pólya and P. Turan, J. Analyse Math. 6 (1958), 323-332.

11. T. Kövari, On the Borel exceptional values of lacunary integral functions, J. Analyse Math. 9 (1961), 71-109.

12. T. Kövari, On the maximum modulus and maximum term of functions analytic in the unit disc, J. London Math. Soc. 41 (1966), 129-137.

13. J. E. Littlewood, Lectures on the theory of functions (Oxford, 1944).

14. P. C. Rosenbloom, Probability and entire functions, Studies in Mathematical Analysis, edited by G. Szegö and others (Stanford University, 1963).

15. W. Saxer, Über die Picardschen Ausnahmewerte sukzessiver Derivierten. Math. Zeit. 17 (1923), 206-227.

16. L. E. Sons, An analogue of a theorem of W. H.J. Fuchs on gap series, Proc. London Math, Soc. (3) 21 (1970), 525-539.

17. E. C. Titchmarsh, The theory of functions, 2nd edition (Oxford, 1939).

18. G. Valiron, Sur les fonctions entières d'ordre fini et d'ordre nul, et en particulier les fonctions à correspondance regulière, Ann. Fac. Sci. Univ. Toulouse (3) 5 (1914), 117-257.

19. G. Valiron, Sur le maximum du module des fonctions entières, C.R. de l'Acad. des sciences (Paris) 166 (1918), 605-608.

20. G. Valiron, Lectures on the general theory of integral functions (reprinted, Chelsea, 1949).

21. G. Valiron, Fonctions entières d'ordre fini et fonctions méromorphes (L'enseignement mathématique, Geneva, 1960).

22. A. Wiman, Über den Zusammenhang zwischen dem Maximalbetrage einer analytischen Funktion und dem grössten Gliede der zugehörigen Taylorschen Reihe, Acta Math. 37 (1914), 305-326.

23. A. Wiman, Über den Zusammenhang zwischen dem Maximalbetrage einer analytischen Funktion und dem grössten Betrage bei gegebenem Argumente der Funktion, Acta Math. 41 (1916), 1-28.

\author{
IMPERIAL COLLEGE \\ LONDON SW 7 \\ ENGLAND
}

